\title{
EXTRACTING UNIAXIAL RESPONSES OF SINGLE CRYSTALS FROM SHARP AND SPHERICAL HARDNESS MEASUREMENTS
}

\author{
J. Alcalá ${ }^{1}$, D. Esqué-de los Ojos ${ }^{1,2}$ and J. Očenášek ${ }^{3}$ \\ ${ }^{1}$ GRICCA, Universitat Politècnica de Catalunya, EUETIB and ETSEIB \\ Barcelona 08028, Spain \\ ${ }^{2}$ Laboratory of Mechanics of Materials \& Nanostructures, EMPA Mat. Sci. \& Technology, \\ $\mathrm{CH}-3602$ Thun, Switzerland \\ ${ }^{3}$ New Technologies Research Centre, University of West Bohemia in Pilsen \\ 30614 Plzeň, Czech Republic
}

\begin{abstract}
This investigation provides a mechanistic background to mechanical property extractions performed from single-crystal microhardness measurements. The analysis concerns both spherical and sharp (pyramidal) indentations. We show that the uniaxial stress-strain curves inferred from hardness measurements in single crystalline units of material are coincidental with those attained under true uniaxial loadings along specific crystalline orientations. Landmark hardness relations that were originally developed for power-law strain hardening polycrystalline aggregates are extended to single-crystal indentations. Mechanical property extractions in crystals violating powerlaw hardening because of a marked critical resolved shear stress and/or extreme strain hardening saturation are subsequently addressed. The analysis is pertinent to the assessment of multipleglide deformation stage-II and strain hardening saturation stage-III of cubic single crystals from indentation experiments.
\end{abstract}

Submitted to Mechanics of Materials

May 2014

\footnotetext{
${ }^{1}$ To whom all correspondence should be addressed. e-mail: jorge.alcala@upc.edu
} 


\section{Introduction}

A central line of work in the analysis of microindentation experiments concerns extraction of the uniaxial stress-strain curve of a small material volume. A fundamental limitation in such mechanical property extractions is that well-established classical correlations between the indentation hardness and the uniaxial behavior concern polycrystalline aggregates (e.g., Hill et al., 1989; Giannakopoulos et al., 1994; Biwa and Störakers, 1995; Dao et al., 2001; Larson, 2001; Mata et al., 2002; Casals and Alcalá, 2005). Therefore, our current knowledge is strictly limited to the analysis of indentation experiments where the imprint encompasses a number of randomly oriented crystals in a polycrystal, rather than to the more relevant case in microindentation testing where the imprint is embedded within a single crystalline unit.

Dislocation gliding in single crystals may become active along a single slip system, resulting in mild hardening (stage-I), or in multiple systems where marked strain hardening is favored (stage-II). As the latter prevail during indention testing, this tool can be potentially used to assess the cross-hardening (multiple-glide) behavior of the crystal as well as its strain hardening saturation response occurring at larger strain levels. Continuum crystal plasticity has become a powerful scheme in describing single-crystal deformation responses under a variety of loading configurations including indentation (e.g., Eidel and Gruttmann, 2007; Liu et al., 2008; Alcalá et al., 2008; Casals and Forest, 2009; Chang et al, 2010; Zambaldi and Raabe, 2010; Saito

et al., 2012; Alcalá and Esqué-de los Ojos, 2012). Hence, this modeling framework could be instrumental in the development of novel methodologies for the assessment of single-crystal uniaxial responses from microhardness measurements.

In addition, continuum mechanics analyses of material size-effects in microindentation experiments can be performed through non-local plasticity models that represent natural extensions of strain-gradient plasticity (e.g., Abu Al-Rub, 2007; Faghihi and Voyiadjis, 2012). Such size effects become experimentally manifest as an increase in hardness at smaller applied 
loads. The analyses account for both statistically stored dislocations, SSDs, and geometrically necessary dislocations, GNDs, where the latter are computed from the strain gradients using Nye's lattice curvature tensor (Evers et al., 2004; Kysar et al., 2007). The results are not however pertinent to the extraction of the uniaxial stress-strain curve of the indented crystal because strain gradients are essentially absent under uniaxial pulling. Moreover, experimental evidences appear to be available indicating that (i) the GND density does not account for the increase in hardness at small scales (Kiener et al., 2006; Rester et al., 2008(a); Rester at al., 2008(b); McLaughlin and Clegg 2008; Demir et al., 2009) and (ii) measured size-dependent spherical indentation responses defy analyses from strain-gradient plasticity (Spary et al., 2006; Zhu et al., 2008). A fundamental understanding to indentation experiments can also be gained through molecular dynamics simulations. These simulations however concern nanoscopic indenter tips, where the inception of plasticity is fundamentally associated with twin/dislocation nucleation phenomena at extremely large hardnesses (see, e.g., Ward et al., 2009; Chang et al., 2010; Engels et al., 2012; Alcalá et al., 2012). Such incipient plasticity responses are not therefore pertinent to the majority of microindentation experiments, where plastic flow is due to mobilization and multiplication of preexisting dislocations.

The main objective of this work is to investigate whether the uniaxial stress - strain curve of a cubic crystal along specific orientations is coincidental with that inferred from hardness measurements. Based on these results, we intent to furnish a mechanistic background extending classic contact mechanics interpretations to hardness testing into the extraction of single-crystal deformation stages-II and III. In doing so, we employ computational simulations with continuum crystal plasticity in conjunction with analyses performed under the $J_{2}$-flow plasticity theory for a coarse-grained isotropic polycrystal. 


\section{Material models and computational simulations}

\subsection{Continuum crystal-plasticity}

This work is lain upon crystal plasticity models providing parallels with the limiting rigid-power-law strain-hardening (Section 2.2) and elastic-perfectly-plastic (Section 2.3) material behaviors, which have been extensively used in contact mechanics interpretations of polycrystalline indentation experiments.

Crystal plasticity simulations rely on Schmid's law to govern onset of plastic flow. The following rate-dependent formulation for the shear strain rates is therefore assumed in this work

$$
\dot{\gamma}^{(\alpha)}=\operatorname{sign}\left(\tau^{(\alpha)}\right) \dot{a}\left|\frac{\tau^{(\alpha)}}{\tau_{\mathrm{c}}^{(\alpha)}}\right|^{m},
$$

where $\tau_{\mathrm{c}}^{(\alpha)}$ is the instantaneous critical shear strength triggering dislocation glide in any given set of the $12\{111\}\langle 1 \overline{1} 0\rangle$ slip systems $(\alpha=1$ to 12$) ; \tau^{(\alpha)}$ and $\dot{\gamma}^{(\alpha)}$ are the shear stress and shear strain rates, respectively, acting on that set of active systems; and $\dot{a}$ and $m$ are material

constants at fixed temperature. The strain hardening law, dictating the increase in $\tau_{\mathrm{c}}^{(\alpha)}$ with deformation, takes the form (Asaro, 1983)

$$
\dot{\tau}_{\mathrm{c}}^{(\alpha)}=\sum_{\beta} h_{\alpha \beta} \dot{\gamma}^{(\beta)}
$$

where $h_{\alpha \beta}$ is the hardening matrix describing the interaction between dislocations gliding in primary $(\alpha)$ and secondary or forest $(\beta)$ systems, and $\dot{\tau}_{\mathrm{c}}^{(\alpha)}$ is computed from the initial critical shear strength $\tau_{\circ}$ producing dislocation glide in the crystal. Matrices $h_{\alpha \beta}$ used in the present investigation are described in Sections 2.2 and 2.3 along with the associated stress-strain curves.

A review of the kinematics of crystalline deformation can be found in Asaro and Rice (1977). First, the deformation gradient $\mathbf{F}$ is decomposed into plastic $\mathbf{F}^{\mathrm{p}}$ and composite elastic and residual lattice rotation $\mathbf{F}^{\star}$, so that

$$
\mathbf{F}=\mathbf{F}^{\mathrm{p}} \cdot \mathbf{F}^{\star}
$$


Velocity gradient tensor $\mathbf{L}$ is then given by

$$
\mathbf{L}=\dot{\mathbf{F}} \cdot \mathbf{F}^{-1}=\mathbf{D}+\boldsymbol{\Omega}=\left(\mathbf{D}^{\mathbf{p}}+\mathbf{D}^{\star}\right)+\left(\mathbf{\Omega}^{\mathbf{p}}+\mathbf{\Omega}^{\star}\right)
$$

where $\mathbf{D}$ is the rate of deformation tensor and $\boldsymbol{\Omega}$ is the rate of spin tensor, both decomposed as in the above, and superscript $\star$ denotes axes that follow crystalline rotation. It is then found that

$$
\mathbf{D}^{\mathrm{p}}+\boldsymbol{\Omega}^{\mathrm{p}}=\sum_{\alpha=1}^{12} \dot{\gamma}^{(\alpha)} \mathbf{s}^{\star(\alpha)} \mathbf{m}^{\star(\alpha)},
$$

where $\mathbf{s}^{\star(\alpha)}=\mathbf{F}^{\star} \cdot \mathbf{s}^{(\alpha)}$ is the slip direction vector and $\mathbf{m}^{\star(\alpha)}=\mathbf{m}^{(\alpha)} \cdot \mathbf{F}^{\star-1}$ is the slip plane vector both following crystalline stretching and rotation.

The Kirchhoff stress tensor $\boldsymbol{\tau}$ and the Jaumann rate of this tensor $\stackrel{\nabla}{\boldsymbol{\tau}}$ are used in the formulation of large-strain theories. Hence, the early elastic response obeys $\stackrel{\nabla}{\boldsymbol{\tau}} \boldsymbol{}=\mathbf{C}: \mathbf{D}^{\star}$ where $\mathbf{C}$ is the elasticity tensor. Following the derivations in Asaro and Rice (1977), the increase in critical shear strength is finally given by

$$
\dot{\tau}_{\mathrm{c}}^{(\alpha)}=\mathbf{m}^{\star(\alpha)} \cdot\left[\nabla_{\boldsymbol{\tau}}^{\star}-\mathbf{D}^{\star} \cdot \boldsymbol{\tau}+\boldsymbol{\tau} \cdot \mathbf{D}^{\star}\right] \cdot \mathbf{s}^{\star(\alpha)} .
$$

\subsection{Hardening models for pure fcc single crystals}

The Bassani and Wu (BW) hardening model (Bassani and Wu, 1991) and the modified version to this model (Alcalá et al., 2008) were used to simulate the indentation response of pure fcc crystals with vanishing critical resolved shear stress $\tau_{\circ}$. The prime differences between the two BW-based models are that (i) the modified version predicts experimentally measured responses for pure copper crystals -see the experiments in Franciosi, 1985, overimposed in Fig. 1(a) for uniaxial loadings along symmetric orientations- and (ii) the original model leads to a much stronger reduction in hardening rate passed a small plastic strain level. Under the strong multiple-glide conditions that prevail during indentation testing (where stage-I is lacking), these models are single-crystal analogs to the rigid-power-law strain hardening material representation at different hardening rates. [The reader is directed to Figs. 4 and 5 for the details of the stress-strain curves in numerous orientations including single glide conditions.] 
A concise description of the models is given in the following. In both BW models, the hardening matrix adopts the form

$$
h_{\alpha \alpha}=\left\{\left(h_{\circ}-h_{\mathrm{I}}\right) \operatorname{sech}^{2}\left[\frac{\left(h_{\circ}-h_{\mathrm{I}}\right) \gamma^{(\alpha)}}{\left(\tau_{\mathrm{s}}-\tau_{\circ}\right)}\right]+h_{\mathrm{I}}\right\}\left\{1+\sum_{\beta \neq \alpha} f_{\alpha \beta} \tanh \left(\frac{\gamma^{(\beta)}}{\gamma_{\circ}}\right)\right\} ; h_{\alpha \beta}=0
$$

where the parameters used in the original model are given in Table 2 from Alcalá et al., 2008. In Eq. (7), the first term at the right hand of $h_{\alpha \alpha}$ accounts for self-hardening and the second term at the right hand incorporates cross-hardening where the sum is performed for slip systems $\beta \neq \alpha$. The first term rapidly approaches $h_{\mathrm{I}}$ with increasing shear strain $\gamma^{(\alpha)}$. Subsequently, the hyperbolic tangent in the second term governs the initiation of cross-hardening, marking the onset of deformation stage-II. Another important feature in Eq. (7) is matrix $f_{\alpha \beta}$ that measures the relative strength of dislocation interactions.

Eq. (7) was revisited in the modified version of the BW model, providing a more robust background to investigate plastic deformation in pure fcc metals (Alcalá et al., 2008; Alcalá and Esqué-de los Ojos, 2012). Adjustments were introduced involving the self-hardening (first) and cross-hardening (second) terms in Eq. (7). In doing so, a stronger connection was stablished between the increase in shear stresses in slip system $\alpha$ and the increase in dislocation density in all secondary $\beta$ systems where the dislocations act as forest obstacles. The following relations were advocated

$$
f_{\alpha \beta}=\left(\frac{\kappa_{\alpha \beta} \ln \left(\rho^{(\beta)^{-1 / 2}} / b\right)}{\kappa_{\alpha \alpha} \ln \left(\rho^{(\alpha)^{-1 / 2}} / b\right)}\right)^{2}
$$

and

$$
h_{\mathrm{I}}=\frac{\mu}{2 K}\left[\kappa_{\alpha \alpha} \ln \left(b \sqrt{\rho^{(\alpha)}}\right)\right]^{2}
$$

where $\rho^{(\beta)}$ is the dislocation density of the forest slip systems, $\mu$ is the shear modulus, $b$ is the Burger's vector and matrix $\kappa_{\alpha \beta}$ gives the strength of the different junctions that arise because of dislocation interactions (e.g., see Kubin et al., 2008). Updating of dislocation densities in 
slip systems $\alpha=1$ to 12 is then performed through (Tabourot et al., 1997)

$$
\dot{\rho}^{(\alpha)}=\frac{1}{b}\left(\frac{\sqrt{\sum_{\beta} a_{\alpha \beta}^{\prime} \rho^{(\beta)}}}{K}-2 y_{\mathrm{c}} \rho^{(\alpha)}\right) \dot{\gamma}^{(\alpha)} .
$$

All parameters in the modified BW model are reported in Table 3 from Alcalá et al., 2008.

\subsection{Hardening models for alloyed fcc single crystals}

The model by Peirce, Asaro and Needleman (PAN) was also used in this work, where it is taken to represent the single-crystal equivalent to the elastic-plastic material description gradually approaching the limit of perfect plasticity (i.e., negligible hardening). This model reproduces the uniaxial behavior of aluminum-copper crystals that exhibit non-vanishing critical resolved shear stress $\tau_{\circ}$ (Peirce et al., 1982). The hardening matrix is written as

$$
h_{\alpha \alpha}=h(\gamma)=h_{\circ} \operatorname{sech}^{2}\left|\frac{h_{\circ} \gamma}{\tau_{\mathrm{s}}-\tau_{\circ}}\right| ; \quad h_{\alpha \beta}=q h_{\alpha \alpha}
$$

where

$$
\gamma=\sum_{\alpha} \int\left|\dot{\gamma}^{(\alpha)}\right| d t .
$$

The parameters given in Table 1 from Alcalá et al., 2008 were used in the present simulations with the PAN model.

The main features from this model are summarized in that deformation stage-I is unaccounted in Eq. (11), where a strong decay in hardening rate setting deformation stage-III is also prescribed by the hyperbolic secant (Fig. 1(b)). Parameter $q$ is key in governing strain hardening under multiple glide. Cross (latent) hardening vanishes at $q=0$, latent and selfhardening become the same at $q=1$, and latent-hardening finally prevails at $q>1$. Hence, $q$ $=0$ sets perfect-plasticity in the interaction of multiple slip systems. At small uniaxial plastic strains $(\epsilon<0.04$ in Fig. 1(b)), the PAN model exhibits a stage-II behavior that consequently weakens as $q \rightarrow 0$. Also, when pulled in asymmetric orientations, strain hardening rapidly saturates as crystalline rotation takes place. It is finally noted that the computed stress-strain curves with the PAN model at $q=1.2$ are within reasonable agreement with the experimental 
measurements in Carlsen and Honeycombe, 1954, for a fully-hardened Al-3.5\% Cu containing $\theta^{\prime}$ precipitates.

Direction attention to Figs. 1(a) and (b), a the remarkable difference between the BW and the PAN models in the context of this investigation is that the critical resolved shear stress $\tau_{\circ}$ essentially vanishes in the former whereas a marked value of $\tau_{\circ}=69 \mathrm{MPa}$ is assumed in the latter. In addition, strain-hardening saturation in stage-III approaches perfect-plasticity in the PAN model whereas a sustained (non-vanishing) hardening rate prevails in the modified version of the BW model depending on crystalline orientation. It is worth noting that while the original BW model does not capture any particular experiments in the literature, its use is instrumental here as it provides an intermediate strain hardening saturation response to that from the modified version of the model and the PAN formulation.

\subsection{Polycrystalline plasticity}

The $J_{2}$-flow plasticity theory was employed in modeling polycrystalline indentation. Such a coarse-grained plasticity theory is suitable in reproducing the indentation behavior of an isotropic polycrystalline aggregate. The simulations are thus taken to reproduce experimental indentation responses where the imprint probes a sufficiently large number of grains. Following McMeeking and Rice (1975), the large-strain formulation of the Prandtl-Reuss equations in the $J_{2}$-flow theory can be written as

$$
\stackrel{\nabla}{\boldsymbol{\tau}}=\mathbf{C}: \mathbf{D}-\left(\left(\frac{9 G^{2}}{3 G+\left(\left(E \cdot E_{\mathrm{T}}\right) /\left(E-E_{\mathrm{T}}\right)\right)}\right) \frac{\mathbf{S}: \mathbf{D}}{\sigma_{\mathrm{e}}^{2}}\right) \mathbf{S}
$$

where $\mathbf{S}=\boldsymbol{\sigma}-1 / 3(\mathbf{I}: \boldsymbol{\sigma}) \cdot \mathbf{I}$ is the stress deviator, $\boldsymbol{\sigma}$ is the Cauchy stress tensor, $\sigma_{e}=$

$\sqrt{3 / 2(\mathbf{S}: \mathbf{S})}$ is the Von Mises stress, $G$ is the shear modulus $\left(G=\frac{E}{2(1+\nu)}\right), E$ is the Young's modulus, $\nu$ is the Poisson ratio, and tangent modulus $E_{T}$ is the instantaneous slope of the true uniaxial stress $(\sigma)$-total true uniaxial strain $(\epsilon)$ curve. 
The piecewise elastic-power law plastic relation was further assumed

$$
\epsilon= \begin{cases}\sigma / E, & \text { if } \sigma<\sigma_{\mathrm{ys}} \\ \left(\sigma / \sigma_{\circ}\right)^{1 / n} & \text { otherwise }\end{cases}
$$

where $\epsilon$ is the sum of elastic and plastic uniaxial strains $\epsilon_{\mathrm{e}}$ and $\epsilon_{\mathrm{p}}$, respectively; and $n$ is the power-law strain hardening coefficient. Invoking continuity between the elastic and plastic branches of Eq. (14), $\sigma_{\circ}$ correlates with yield strength $\sigma_{\mathrm{ys}}$ through

$$
\sigma_{\circ}=E^{n} \sigma_{\mathrm{ys}}^{1-n}
$$

Notice that the generic form $\sigma=\sigma_{\circ} \epsilon^{n}$ in Eq. (14) also describes rigid-strain hardening responses in the absence of linear elasticity, where Eq. (15) is rendered invalid as $\sigma_{\mathrm{ys}} \rightarrow 0$.

\subsection{Finite element simulations}

Finite element (FE) simulations were performed for spherical and pyramidal (Berkovich) indentations of polycrystals and single-crystals with the (001), (011) and (111) surface orientations. The three-dimensional cylindrical mesh whose cross-sectional view is shown in Fig. 2 was used in all simulations. The mesh contains 11500 brick-shape eight-noded elements and is composed by 8 regions of different element size, where the smaller elements are placed at the contact region and its proximities. The mesh can slide freely against the bottom flat surface during indentation. The outer free surfaces in the radial and vertical directions are always maintained at a distance to the imprint's center greater than 25 times the contact radius. Two spherical tips whose diameters $D$ differ by a factor of two were employed in the computation of the hardness evolutions with increasing penetration at $a / D<0.10$ (large $D$ ) and $a / D>0.10$ (small $D$ ), where $a$ is the contact radius. Moreover, by converging at $\Delta \gamma^{(\alpha)} \leq 1.2 \times 10^{-4}$ in each time increment for all material points, the computational fluctuation was reduced to less than $5 \%$ in hardness. This enabled hardness measurements at deep penetrations well exceeding $a / D=0.20$.

The uniaxial stress-strain curves were modeled with a single brick-shape element. The 
bottom surface of the element was allowed to slide and to rotate freely along a fixed nodal point during the application of uniform displacement at its top surface. The simulations, performed for each of the crystal plasticity models, were conducted by systematically orienting the element along 231 locations within the Lambert azimuthal equal-area projection.

\section{Discussion}

\subsection{Extracting mechanical properties from single-crystal spherical hardnesses}

Mechanical property extractions from polycrystalline spherical indentations have long been based on the landmark hardness relation by Tabor (e.g., Tabor, 1951)

$$
\bar{p}=P / A=2.8 \sigma_{\circ}\left(0.4 \frac{a}{D}\right)^{n}
$$

where $\bar{p}$ is the hardness, $a$ is the contact radius, $D$ is the diameter of the indenter, projected contact area $A=\pi a^{2}, P$ is the applied indentation load, and $\sigma_{\circ}$ and $n$ are the sought-after uniaxial plastic properties of the material (Eq. (14)). A theoretical background for Eq. (16) was provided by Hill et al., (1989) based on plastic-similarity analyses, where the strict values of 2.8 and 0.4 were further found through $J_{2}$-flow FE simulations (Biwa and Störakers, 1995; Alcalá and Esqué-de los Ojos, 2010). Since linear elasticity is neglected in Eq. (16), this relation becomes the asymptotic bound for fully-plastic indentations performed in isotropic polycrystalline aggregates. These polycrystals fulfill general power-law hardening $\sigma=\sigma_{\circ} \epsilon^{n}$, where the correlation in Eq. (15) is obviously invalid in the limit of $\sigma_{\mathrm{ys}} \rightarrow 0$.

Irrespectively of surface orientation, Eq. (16) fits the hardness evolutions from the BW models given in Fig. 3(a). The $n$ value obtained from the slope of the $\ln \bar{p}$ vs $\ln (a / D)$ plot along with the value of $\sigma_{\circ}$ measured from the intersection of this logarithmic representation with the $\ln \bar{p}$ (vertical) axis are given in Table 1 . It is noticed that the entire hardness evolutions from small to large levels of $a / D$ are fitted with unique values of $n$ and $\sigma_{\circ}$. Moreover, Fig. 3(a) illustrates that the crystal plasticity simulations provide close bounds to the experimental 
results by Lim and Chaudhri (1999) for pure copper.

The hardness evolutions from the PAN model are also insensitive to surface orientation (Fig. 3(b)). Neverthless, the values of $n$ and $\sigma_{\circ}$ inferred from Eq. (16) vary depending on the range of $a / D$. For $0.07<a / D<0.13, n=0.2$ and $\sigma_{\circ}=590 \mathrm{MPa}$ are found from the above logarithmic data fit (Table 1). This is due to the fact that at $a / D<0.07$, Eq. (16) is rendered invalid in materials exhibiting a marked $\sigma_{\mathrm{ys}}$. By drawing a parallel between the role of $\sigma_{\mathrm{ys}}$ in polycrystalline indentation and the critical resolved shear stress $\tau_{\circ}$ in single-crystal indentations, an elasto-plastic (transitional) regime is therefore anticipated to develop in single crystals with marked $\tau_{\circ}$ indented at small levels of $a / D$. The fully-plastic regime may then rule the indentation response only at a sufficiently large $a / D$. Large values of $\tau_{\circ}$ would enlarge the range of $a / D$ for the elasto-plastic transition while small values of $\tau_{\circ}$ would shrink such a range. The general methodology proposed in Flow Chart 1 from Alcalá and Esqué-de los Ojos (2010) is thus pertinent here, as it enables mechanical property extractions in both the transitional elasto-plastic and the fully-plastic regimes. For the PAN model at $q=1.2$ and $a / D<0.13$, the methodology provides nearly identical values of $n$ and $\sigma_{\circ}$ as those reported above for the fully-plastic range of $0.07<a / D<0.13$. Table 1 also gives the inferred properties from this methodology for the PAN model with $q=0(a / D<0.07$ in Fig. 3(b)). As expected, $q \rightarrow 0$ produces negligible cross-hardening, so that the inferred $n$ decreases from $n=0.2$ for $q=1.2$ to $n=0.1$ for $q=0$.

Perfectly-plastic polycrystals with $n=0$ deforming through $J_{2}$-flow plasticity exhibit a gradual decrease in hardness with increasing $a / D$ (Alcalá and Esqué-de los Ojos (2010)). Similar results are found for the PAN model, indicating that passed a certain penetration of the spherical tip, the contact response is dominated by the strain hardening saturation stage-III behavior in the crystal (see Fig. 3(b) for $a / D>0.07$ at $q=0$ and $a / D>0.13$ at $q=1.2$ ). Such an indentation behavior is thus amenable to analysis through (Alcalá and Esqué-de los 
Ojos (2010))

$$
\bar{p} / \sigma_{\mathrm{s}}=-1.885 a / D+3.044
$$

where $\sigma_{\mathrm{s}}$ is the uniaxial (cut-off) stress marking strain hardening saturation (perfect-plasticity). Linear fitting of the decreasing hardness evolutions from the PAN model in Fig. 3(b) yields $\sigma_{\mathrm{s}}$ $=360 \mathrm{MPa}$ and $215 \mathrm{MPa}$ for $q=1.2$ and 0 , respectively (Table 1 ).

The experimental scatter in spherical microindentations performed in the present sizeindependent length scale (i.e., for $D>400 \mu \mathrm{m}$ ) may be as large as $\pm 30 \%$ for $a / D<0.05$ (mechanically polished surfaces with colloidal silica finish). Experimental variability however decreases to less than, say, $\pm 5 \%$ at greater normalized penetrations (Lim and Chaudhri, 1999). By performing more than 10 hardness measurements throughout the extended range of $0.05<$ $a / D<0.4$, the variability in the inferred mechanical properties from the logarithmic data fit to Eq. (16) can be reduced. One shall also bear in mind that when large values of $n$ are extracted from this logarithmic representation, the associated $\sigma_{\circ}$ exhibits greater statistical fluctuations. Analysis of the experimental scatter for 10 hardness measurements $(a / D>0.05)$ indicates that the inferred $n$ may fluctuate in $\pm \sim 0.05$ for $n>0.5$; in $\pm \sim 0.04$ for $0.5>n>0.3$; and in $\pm \sim 0.02$ for $0.3>n>0.1$

\subsection{Extracting mechanical properties from single-crystal pyramidal hardnesses}

From Tabor's work, the following fully-plastic hardness bound is the equivalent of Eq. (16) for sharp (pyramidal) polycrystalline indentations (Tabor, 1951)

$$
\bar{p}=C \sigma_{0.1},
$$

where $\sigma_{0.1}$ is the uniaxial stress of the indented material at a fixed (representative) uniaxial strain of $\epsilon=0.10$. Power-law hardening then readily yields $\sigma_{0.1}=\sigma_{\circ}\left(0.1^{n}\right)$. As in the case of spherical indentations, the mathematical structure of Eq. (18) emerges from plastic-similarity analyses (Larsson, 2001). FE simulations with the $J_{2}$-flow theory then provide $C=2.60$ for 
pyramidal Berkovich indentation (Casals and Alcalá, 2005), where the fully-plastic bound in Eq. (18) is reached for $E / \sigma_{0.1}>110$.

By again drawing a parallel between polycrystalline and single-crystal indentations, the simulated hardnesses from the different crystal plasticity models are inputted in Eq. (18) to extract values for $\sigma_{0.1}$ (Table 2). As $E / \sigma_{0.1}>110$, onset of full plasticity is verified with the inferred properties in Table 1. [When indenting harder (elasto-plastic) crystals with $E / \sigma_{0.1}<110$, parameter $\sigma_{0.1}$ can be inferred from the general hardness relation from Casals and Alcalá, 2005 (Eq. A.1 for Vickers and Berkovich indentations) in conjunction with the figures provided in Hernot et al. (2014)].

The experimental scatter in size-independent single-crystal pyramidal hardness measurements performed in mechanically polished surfaces with colloidal silica finish is of $\pm 5 \%$ (Lim and Chaudhri, 2002). This scatter translates directly into the assessment of uniaxial stress $\sigma_{0.1}$ by virtue of Eq. (18).

As the severity of the indentation strains in the subsurface increases with increasing $a / D$, a penetration may be reached where the spherical hardness becomes the same as the pyramidal hardness. The present results show that this condition indeed occurs for $a / D \approx 0.25$ (compare the Berkovich hardness in Table 2 with the spherical hardness at $a / D \approx 0.25$ in Fig. 3 for the different crystal plasticity models). This is a useful result that facilitates extraction of single crystal properties from microindentation experiments, where material size effects cease to occur at shallower penetrations with sharp tips than with spherical counterparts. In the event where microindentations are performed in polycrystalline aggregates, size-independent sharp microcontacts are more likely to occur at smaller imprint sizes thus lying within a single grain (i.e., without intervention of grain boundary strengthening). From the above results, it follows that such single crystal pyramidal hardness would be coincidental with a spherical hardness value at $a / D \approx 0.25$ hypothetically obtained in the absence of both indentation size-effects and grain boundary strengthening. 


\subsection{The finding of representative uniaxial orientations}

The mechanical properties inferred in Sections 3.1 and 3.2 lack of any fundamental physical meaning. This is because (i) single-crystal indentations are analyzed using hardness relations that are strictly valid for isotropic polycrystals and (ii) cross-hardening depends on the orientation subjected to uniaxial loading, and is conceivably stronger under indentation testing where the 12 slip systems become simultaneously active (Alcalá and Esqué-de los Ojos, 2010). While the former issue involves limitations in the assumed constitutive response, the latter concerns the essential debate of whether contact loadings in single crystals can be truly examined under the basis of simple uniaxial loading responses. The following discussion remarkably shows that the stress-strain curve along the plastically stiffest [111] orientation represents an upper bound to the uniaxial behavior inferred from single-crystal indentations.

Figs. 4-7 show the extreme sensitivity of the uniaxial stress-strain curves from the BW and PAN models to 231 pulling orientations in the Lambert azimuthal equal-area projection. This is a manifestation of the strong dependency of strain hardening to the development of the different orientation-sensitive dislocation junctions.

With the purpose of correlating the properties inferred from indentation to the uniaxial stress-strain curves along specific loading orientations, we have chosen to compare the values of $\sigma_{0.1}$ from Table 1 (spherical indentation) to those from the simulations under uniaxial loads with the different single-crystal hardening models. In doing so, the above 231 orientations were taken to provide sufficient accuracy as the difference between the value of $\sigma_{0.1}$ in any two neighboring orientations in the equal-area projection is always less than $7 \%$.

Figs. 4-7 and Table 3 summarize the main findings from the above analysis. In the modified BW model (Fig. 4), the marked ellipse of 5 to $7^{\circ}$ misorientations with respect to the [111] pulling axis exhibit less than $10 \%$ variations in the value of $\sigma_{0.1}$ as compared to that inferred through indentation. Similar results hold for the original BW model, where less than $10 \%$ variations between the inferred $\sigma_{0.1}$ and the actual values measured under uniaxial loadings 
happen to occur for the marked ellipse of 4 to $6^{\circ}$ misorientations with respect to the [111] axis (Fig. 5). The good agreement in such loading orientations for the two models holds even though the uniaxial behavior from the original BW formulation undergoes a much more stronger strain hardening reduction (see Fig. 5 (b) at $\epsilon>0.02$ ). Moreover, notice that whereas a pronounced single-crystal deformation stage-I develops for the modified version of the BW model in the above misorientations (see Fig. 4 (b) at $\epsilon<0.015$ ), the subsequent multiple-glide stage-II response rapidly increases hardening rate. Hence, for say $\epsilon>0.03$ in Fig. 4 (b), good accord is readily reached between the actual stress-strain curves and the power-law behavior inferred from indentation.

The stress-strain curves extracted from the PAN model gradually depart from the uniaxial responses along the above misorientations (Figs. 6 and 7). For $q=1.2$, the marked 10 to $15^{\circ}$ misorientations with respect to the [111] axis, that exhibit softer overall stress-strain responses, become in better accord with the inferred behavior from indentation (Fig. 6). An increased tendency for a perfectly-plastic indentation regime is herein probed with the PAN model at $q=0$. In this case, the accordance between the inferred saturation stress $\sigma_{\mathrm{s}}$ and the actual value measured under uniaxial loads further shifts towards the marked ellipse of 25 to $27^{\circ}$ misorientations in Fig. 7.

\subsection{Background to single-crystal mechanical property extractions}

Following the above discussion, mechanical property extractions from single-crystal indentations provide representative stress-strain curves that coincide with the uniaxial behavior of the crystal when pulled along particular sets of crystalline orientations. One shall bear in mind that Tabor's hardness relations (Eqs. (16) and (18)) are sustained upon plastic-similarity interpretations in conjunction with $J_{2}$-flow plasticity $\mathrm{FE}$ simulations. In these analyses, the indented solid exhibits a power-law (homogeneous) relation between the stresses and the strains where linear elasticity is neglected (Hill et al., 1989; Biwa and Störakers, 1995; Larsson, 2001). 
Since Eq. (16) fits the entire hardness evolutions from the BW-models in Fig. 3(a), the above mechanistic rationale can be readily extended to single-crystal indentations.

The symmetry between rigid-power-law plastic polycrystalline and single-crystal indentations thus entails because the BW models are characterized by a vanishing $\tau_{\circ}$, so that linear elasticity can be neglected, and the stress-strain curves fulfill orientation-sensitive power-law functions. Since $J_{2}$-flow FE simulations conducted with the inferred (representative) uniaxial stress-strain curves (Table 1) reproduce the single-crystal hardnesses in Fig. 3(a), accuracy of Eqs. (16) and (18) is confirmed.

In contrast to the BW-models, the PAN formulation describes contacts that violate plasticsimilarity because (i) the representative (inferred) stress-strain curve exhibits marked yield strength (leading to the elasto-plastic indentation regime) and (ii) a disruption of power-law hardening occurs at the inferred cut-off stress $\sigma_{\mathrm{s}}$. When the representative stress-strain curves in Table 1 for the PAN model are used as input for $J_{2}$-flow simulations, the single-crystal hardnesses in Fig. 3(b) are also recovered. [In doing so, the inferred properties are inputted in tabular form including cut-off stress $\sigma_{\mathrm{s}}$ ]. This confirms accuracy of the presently used methodology for mechanical property extractions in the absence of plastic-similarity.

Concerning comparison between the stress-strain curves extracted from spherical and pyramidal indentations with the BW and PAN models, it is noticed that the value of $\sigma_{0.1}$ obtained with both indenter tips vary by an utmost of, say, 15\% (compare Tables 1 and 2). This is a relatively small variation when considering that the plastic shear strain levels underneath the surface are much more greater in pyramidal than in spherical indentations. Consequently, in the case of the PAN model at $q \rightarrow 0$, pyramidal indentation is anticipated to readily probe the strain hardening saturation behavior developing at large strains. This is confirmed as the inferred value for $\sigma_{0.1}$ from Berkovich indentation approaches the saturation (cut-off) value of $\sigma_{\mathrm{s}}$ extracted from spherical indentation (Eq. (17)) at $a / D>0.07$ (compare Tables (1) and (2) for $q=0$ ). This is further illustrative in that the assessment of the strain hardening (stage-II) 
response through spherical indentation in crystals undergoing strong hardening saturation is only feasible at small values of $a / D$.

An interesting conception emerging from this investigation is that the contact response in plastically-similar indentations (such as those developing in high-purity fcc crystals) can be captured through a homogenized description for the plastic shear strains in the slip systems (Eq. (12)). Power-law hardening is then subsequently enforced with a suitable function for $h_{\alpha \beta}(\gamma)$ (see Eq. (11)). We advocate

$$
h_{\alpha \alpha}=h(\gamma)=\kappa \tau_{\circ}^{\star} \gamma^{\kappa-1} ; h_{\alpha \beta}=q h_{\alpha \alpha}
$$

because the substitution of Eq. (19) into the formulation for $\dot{\tau}_{c}^{(\alpha)}$ (Eq. (2)) retains the same mathematical structure as the derivative $\dot{\sigma}=n \sigma_{\circ} \epsilon^{n-1} \dot{\epsilon}$ in the power-law plastic model. In Eq. (19), $\tau_{\circ}^{\star}, \gamma$ and $\kappa$ thus play the role of $\sigma_{\circ}, \epsilon$ and $n$, respectively.

In the above modeling approach, the crystallographic character of the surface topography and of the plastic zone shape are captured by the kinematics of continuum crystal plasticity (Eqs. (3)-(6)). The hardening matrix then dictates the hardness value, the magnitude of the pileup/sinking-in responses of the indented surface, and the accumulation of plastic strains underneath the imprint (Alcalá et al., 2008; Alcalá et al., 2010; Alcalá and Esqué-de los Ojos, 2012). Parameters $\tau_{\circ}^{\star}, \gamma$ and $\kappa$ can thus be adjusted to reproduce a given experimentally measured indentation response. In doing so, one shall bear in mind that the stress-strain curve from the tentative (candidate) crystal-plasticity hardening model along the (111) orientation represents an upper bound to the mechanical behavior of the crystal inferred from indentation experiments. From Section 3.3, it follows that this upper bound is strictly approached at large hardening rates.

\section{Concluding remarks}

Mechanical property extractions from indentation experiments resort to analytical formulations developed for polycrystalline metals, where the imprint surrounds a number of grains or 
crystalline units. This framework is not however pertinent to the majority of microindentation experiments, which probe material responses at microstructural scales where yielding and strain hardening are strongly dependent on crystallographic orientation. This work represents the first attempt to extract single-crystal uniaxial stress-strain responses from spherical and sharp indentation experiments in the absence of material size effects.

The following are the main conclusions drawn from this work.

1. The uniaxial stress-strain curve of a single-crystal pulled in the plastically stiffest [111] orientation represents an upper bound to the mechanical response inferred from indentation. This result is noteworthy because indentation experiments impose extreme multipleglide conditions in the crystal that are distinctly different to those developing under uniaxial loadings. In the case of crystals with vanishing critical resolved shear stress $\tau_{\circ}$ and pronounced strain hardening (BW models), the representative uniaxial behavior inferred from indentation coincides with that of the crystal when pulled along an ellipse of 4 to $7^{\circ}$ misorientations with respect to the [111] direction (Figs. 3 and 4). As $\tau_{\circ}$ is increased and strain hardening becomes less prominent (PAN model with $q \rightarrow 0$ ), the above set of representative misorientations gradually shift towards a limiting ellipse of $25^{\circ}$ with respect to the [111] axis (Fig. 6).

2. A mechanical background for the correlation between indentation and uniaxial responses emerges from the investigation. Guidelines are given in the paper to distinguish between the indentation behavior of crystals with large critical resolved shear stress $\tau_{\circ}$ undergoing mild strain hardening (PAN model), and pure crystals with vanishing $\tau_{\circ}$ and pronounced hardening (BW models). The latter contacts behave in a plastically-similar (fully-plastic) way, so that an early linear-elastic response is absent and the inferred (representative) stress-strain curve fits a power-law function as described by Tabor's relations (Eqs. (16) and (18)). Plastic-similarity is violated with increasing $\tau_{\circ}$, where the early elastic behav- 
ior affects on the measured hardness. Transition from the elasto-plastic contact regime towards full-plasticity is probed by gradually increasing the penetration of a spherical tip in crystals with marked $\tau_{\circ}$ and mild hardening. This has clear consequences to mechanical property extractions including assessment of strain-hardening saturation (stage-III) responses. While spherical indentation at small values of $a / D$ is advocated in probing the early stage-II response in such crystals, assessment of saturation stress $\sigma_{\mathrm{s}}$ becomes accesible from sharp indentation or by employing greater tip penetrations in spherical indentation $(a / D \geq 0.15)$.

3. Whereas sharp and spherical indentation experiments produce different plastic strain levels, a reasonable accord is found in the value of the uniaxial stress at a uniaxial plastic strain of $0.1\left(\sigma_{0.1}\right)$ inferred with both indenter tips. Although at small and large uniaxial strain levels $(\epsilon<0.02$ and $\epsilon>0.15$, respectively) some deviations are found between the stress-strain curves in the aforementioned sets of misorientations and the inferred uniaxial responses, the agreement is optimum at $\sigma_{0.1}$. An equivalency between sharp and spherical hardness measurements is reported at a normalized contact radius of $a / D \approx 0.25$.

Acknowledgment- JA acknowledges financial support by the Spanish Ministry of Science and Innovation through project MAT2011-23375. JO acknowledges support from the CENTEM project CZ.1.05/2.1.00/03.0088.

\section{References}

Abu Al-Rub, R.K., Voyiadjis, G.Z., 2004. Analytical and experimental determination of the material intrinsic length scale of strain gradient plasticity theory from micro- and nanoindentation experiments. Int. J. Plasticity. 20, 1139-1182 .

Abu Al-Rub, R.K., 2007. Prediction of micro and nano indentation size effect from conical or pyramiddal indentation. Mech. Mater. 39, 787-802 . 
Alcalá, J., Casals, O., Ocenasek, J., 2008. Micromechanics of pyramidal indentation in FCC metals: single-crystal plasticity finite element analysis. J. Mech. Phys. Solids. 56, 3277-3303.

Alcalá, J., Esqué-de los Ojos, D., 2010. Reassessing spherical indentation: contact regimes and mechanical property extractions. Int. J. Solids and Struct. 47, 2714-2732.

Alcalá, J., Esqué-de los Ojos, Ocenasek, J., 2010. Continuum crystal plasticity analyses of the plastic flow features underneath single-crystal indentations. Philos. Mag. A, 91, 1050-1069.

Alcalá, J., Esqué-de los Ojos, 2012. Toward the development of continuum single-crystal contact mechanics analyses to microindentation experiments. Comp. Mater. Sci. 52, 14-19.

Alcalá, J., Dalmau, R., Franke, O., Biener, M., Biener, J., Hodge, A., 2012. Planar defect nucleation and annihilation mechanisms in nanocontact plasticity of metal surfaces. Phys. Rev. Lett. 109075502.

Asaro, R.J., Rice, J. R., 1977. Strain localization in ductile single crystals. J. Mech. Phys. Solids 25, 309-338.

Asaro, R.J., 1983. Micromechanics of crystals and polycrystals. Adv. Appl. Mech. 23, 1-115.

Bassani, J.L., Wu, T.Y., 1991. Latent hardening in single crystals. II. Analytical determination and predictions. Proc. R. Soc. London A. 435 21-41.

Biwa, S., Störakers, B.J., 1995. Analysis of fully plastic Brinell indentation. J. Mech. Phys. Solids 43, 1303-1334.

Carlsen, K.M., Honeycombe, R.W.K., 1954. Plastic deformation of aluminum-3.5\% copper alloy single crystals. J. Inst. Met. 83, 449-454.

Casals, O., Alcalá, J., 2005. The duality in mechanical property extractions from Vickers and Berkovich instrumented indentation experiments. Acta Mater. 53, 3545-3561. 
asals, O., Forest, S., 2009. Finite element crystal plasticity analysis of spherical indentation in bulk single crystals and coatings. Comp. Mater. Sci., 45, 774-782.

Chang, H-J., Fivel, M., Rodney, D., Verdier, M., 2010. Multiscale modelling of indentation in FCC metals: From atomic to continuum. C.R. Physique 11, 285-292.

Dao, M., Chollacoop, N., Van Vliet, K.J., Venkatesh, T.A., Suresh, S., 2001. Computational modeling of the forward and reverse problems in instrumented sharp indentation. Acta Mater. 49, 3899-3918.

Demir, E., Raabe, D., Zaafarani, N., Zaefferer, S., 2009. Investigation of the indentation size effect through the measurement of the geometrically necessary dislocations beneath small indents of different depths using EBSD tomography. Acta Mater. 57, 559-569.

Eidel, B., Gruttmann, F., 2007. Squaring the circle - A curious phenomenon of fcc single crystals in spherical microindentation. Comput. Mater. Sci. 39, 172-178.

Engels, P., Ma, A., Hartmaier A., 2012. Continuum simulation of the evolution of dislocation densities during nanoindentation, Int. J. Plasticity 38, 159-169.

Evers, L.P., Brekelmans, W.A.M., Geers, M.G.D., 2004. Non-local crystal plasticity model with intrinsic SSD and GND effects. J. Mech. Phys. Solids 52, 2379-2401.

Faghihi, D., Voyiadjis, G.Z., 2012. Determination of nanoindentation size effects and variable material intrinsic length scale for body-centered cubic metals. Mech. Mater. 44, 189-211.

Franciosi, P., 1985. The concepts of latent hardening and strain hardening in metallic single crystals. Acta metall. 33, 1601-1612.

Hernot, X., Mousa, C., Bartier, O., 2014. Study on the concept of representative strain and constraint factor introduced by Vickers indentation. Mech. Mater. 68, 1-14. 
Giannakopoulos, A.E., Larsson, P.-L., Vestergaard, R., 1994. Analysis of Vickers indentation. Int. J. Solids and Struct. 31, 2679-2708.

Hill, R., Störakers, B., Zdunek, A.B., 1989. A theorical study of the Brinell hardness test. Proc. Royal Soc. London A423, 301-330.

Kiener, D., Pippan, R., Motz, C., Kreuzer, H., 2006. Microstructural evolution of the deformed volume beneath microindents in tungsten and copper. Acta. Mater. 54, 2801-2811.

Kubin, L., Devincre, B., Hoc, T., 2008. Modelling dislocation storage rates and mean free paths in face-centered cubic crystals. Acta Mater. 56, 6040-6049.

Kysar, J.W., Gan, Y.X., Morse, T.L., Chen, X., Jones, M.E., 2007. High strain gradient plasticity associated with wedge indentation into face-centered cubic single crystals: Geometrically necessary dislocation densities. J. Mech. Phys. Solids 55, 1554-1573.

Larsson, PL., 2001. Investigation of sharp contact at rigid-plastic conditions. Int J. Mech. Sci. $43,895-920$.

Lim, Y.Y., Chaudhri, M.M., 1999. The effect of the indenter load on the nanohardness of ductile metals: an experimental study on polycrystalline work-hardened and annealed oxygen-free copper. Philos. Mag. A. 79, 2979-3000.

Lim, Y.Y., Chaudhri, M.M., 2002. The influence of grain size on the indentation hardness of high-purity copper and aluminum. Philos. Mag. A. 82, 2071-2080.

Mata, M., Anglada, M., Alcalá, J., 2002. Contact deformation regimes around sharp indentations and the concept of the characteristic strain. J. Mater. Res. 17, 964-976.

McLaughlin, K.K., Clegg, W.J., 2008. Deformation underneath low-load indentations in copper. J. Phys. D: Appl. Phys. 41, 074007. 
McMeeking, R.M., and Rice, J.R., 1975. Finite-element formulations for problems of large elastic-plastic deformation. Int. J. Solids and Struct. 11, 601-606.

Peirce, D., Asaro, R.J., Needleman, A, 1982. An analysis of nonuniform and localized deformation in ductile single-crystals, Acta Metall. 30, 1087-1119.

Rester, M., Motz, C., Pippan, R., 2008(a). Stacking fault energy and indentation size effect: Do they interact?. Scr. Mater. 58, 187-190.

Rester, M., Motz, C., Pippan, R., 2008(b). The deformation-induced zone below large and shallow nanoindentations: A comparative study using EBSD and TEM. Philos Mag. Lett. 88. $879-887$.

Spary, I.J., Bushby, A.J., Jennett, N.M., 2006. On the indentation size effect in spherical indentation. Philos. Mag. A. 86, 5581-5593.

Tabor, D., 1951. The Hardness of Metals. Clarendon Press, United Kingdom.

Tabourot, L., Fivel, M., Rauch, E., 1997. Generalized constitutive laws for f.c.c. single crystals. Mater. Sci. Eng. A 234-236, 639-642.

Ward D.K., Farkas, D., Lian, J., Curtin, W.A., Wang, J., Kim, K.-S., Qi, Y., 2009. Engineering size-scaling of plastic deformation in nanoscale asperities. Proc. Natl. Acad. Sci. U.S.A., 106, 9580-9585.

Zambaldi, C., Raabe, D., 2010. Plastic anisotropy of $\gamma$-TiAl revealed by axisymmetric indentation. Acta Mater. 58, 3516-3530.

Zhu, T.T., Hou, X.D., Bushby, A.J., Dunstan, D.J., 2008. Indentation size effect at the initiation of plasticity for ceramics and metals. J. Phys. D Appl. Phys. 41, 074004. 
a)

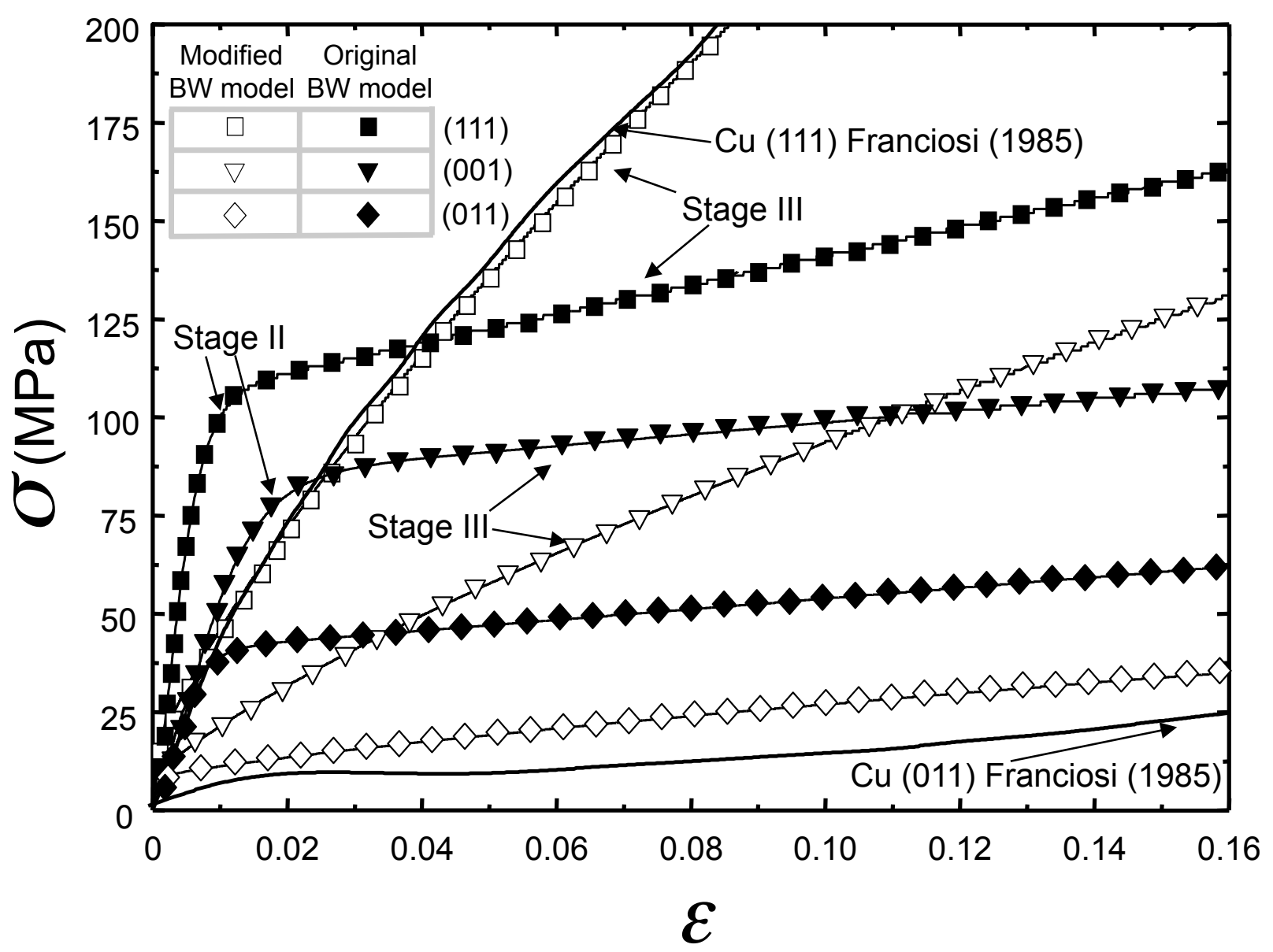

Fig. 1: Uniaxial stress-strain curves in different orientations. Part (a) is for crystals deforming through BW models. These are characterized by a vanishing critical resolved shear stress, $\tau_{o}$ at different strain hardening responses. Part (b) is for crystals deforming through the PAN model with marked $\tau_{o}$ and stronger strain hardening saturation responses. Continuous lines in Parts (a) and (b) are from experiments for pure copper crystals (Franciosi, 1985) and from experiments for Al-3.5\% Cu (Carlsen and Honeycombe, 1954), respectively. Single-crystal deformation stages II and III are marked in the figures. 
b)

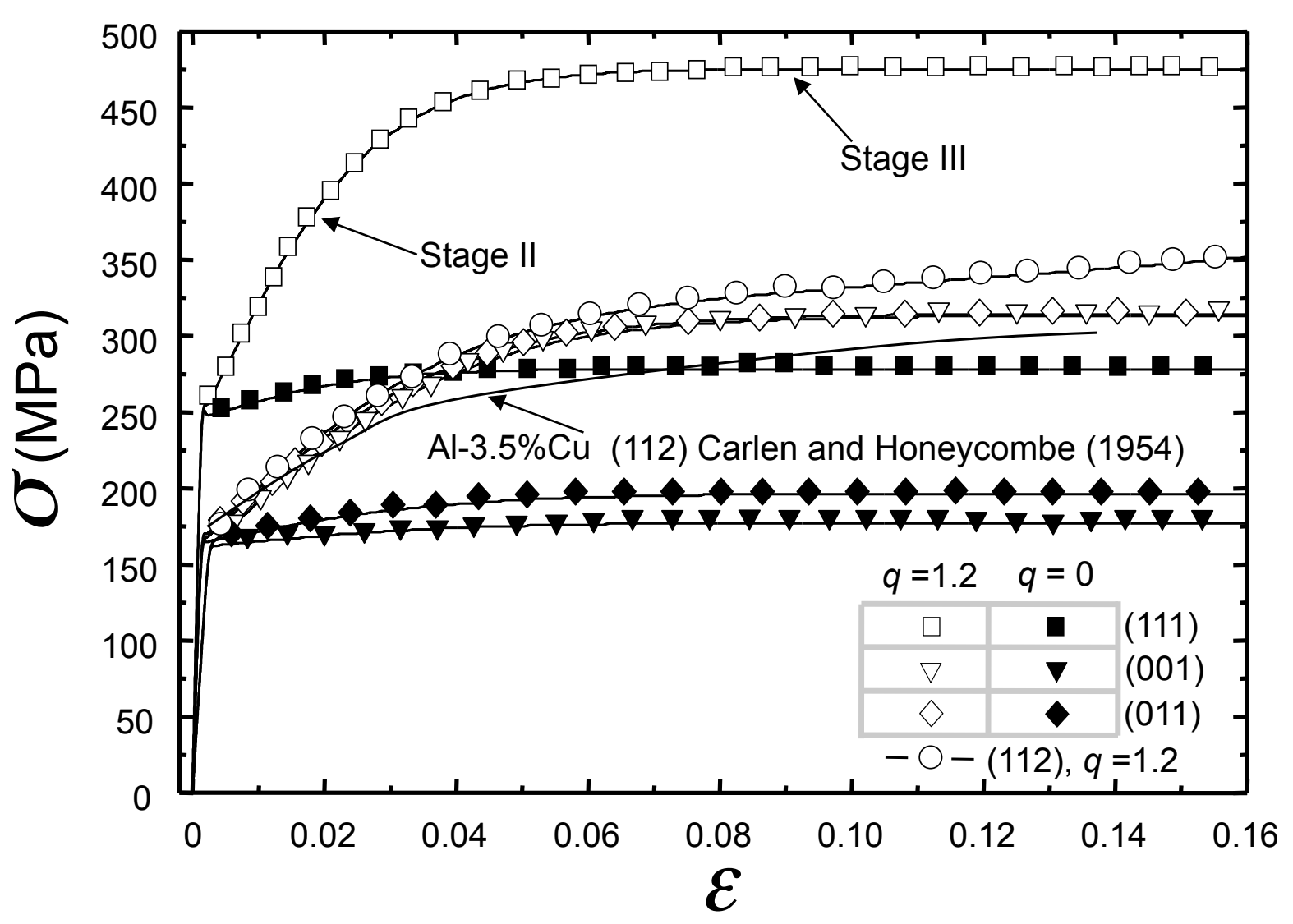

Fig. 1: Uniaxial stress-strain curves in different orientations. Part (a) is for crystals deforming through BW models. These are characterized by a vanishing critical resolved shear stress, $\tau_{o}$ at different strain hardening responses. Part (b) is for crystals deforming through the PAN model with marked $\tau_{o}$ and stronger strain hardening saturation responses. Continuous lines in Parts (a) and (b) are from experiments for pure copper crystals (Franciosi, 1985) and from experiments for Al-3.5\% Cu (Carlsen and Honeycombe, 1954), respectively. Single-crystal deformation stages II and III are marked in the figures. 
Fig. 2

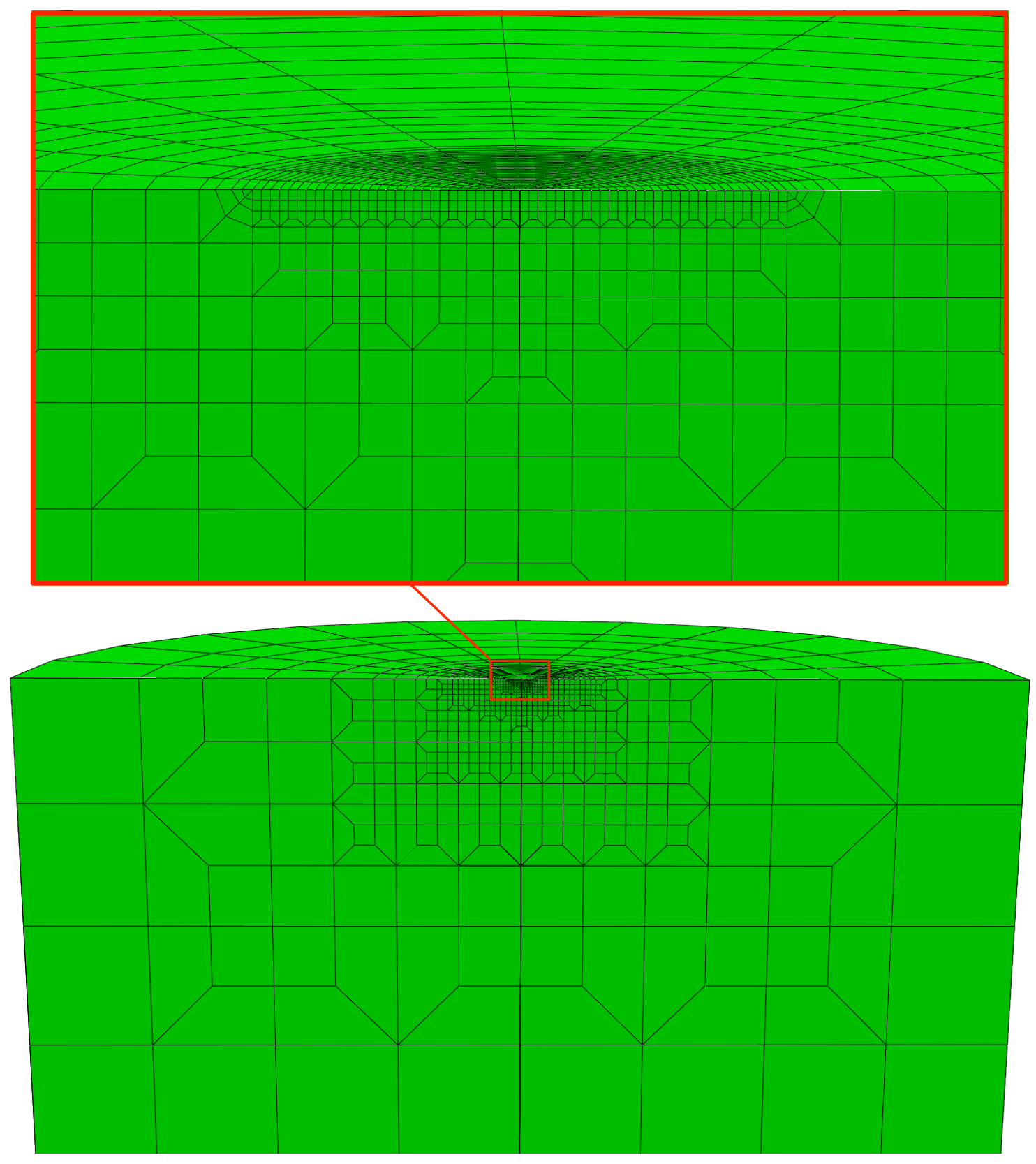

Fig. 2: Cross-sectional view of the finite element mesh employed in the simulations. The indented (central) region is shown in detail. 
a)

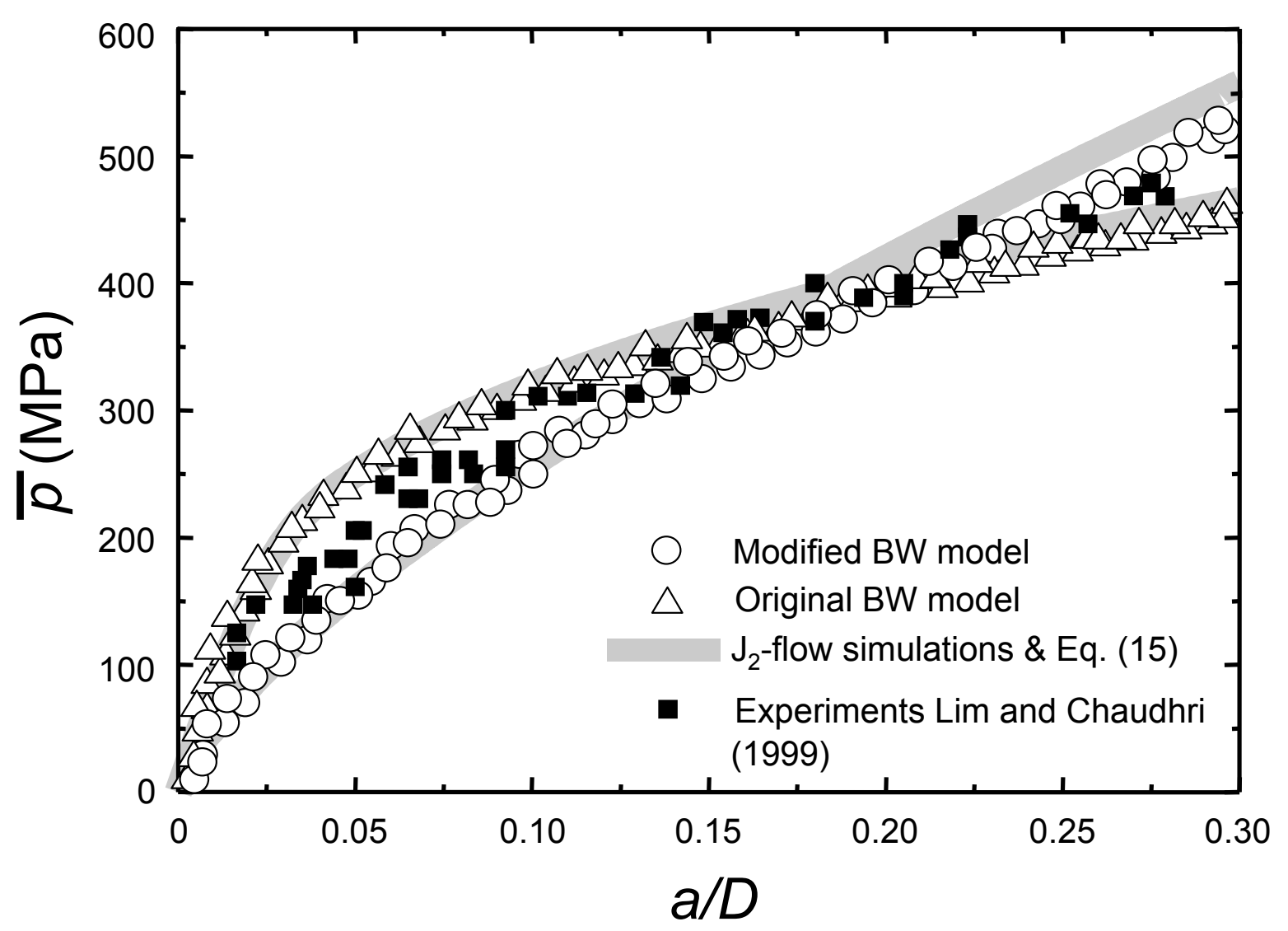

Fig. 3: Evolutions of hardness, $\bar{p}$, with increasing normalized contact radius, $a / D$. Part (a) is for pure copper (BW models), where the crystal plasticity simulations for the (001), (011) and (111) orientations overlap within computational scatter. The indentation behavior is archetypal of that in strong strain hardening crystals where stage-II dominates. Part (b) is for the PAN model where the gradual hardness decrease at $a / D>0.15$ is characteristic of the marked strain hardening saturation response during single-crystal stage-III. Notice that orientation-independent hardnesses are found in Parts (a) and (b). $J_{2}$-flow theory FE simulations are performed with the inferred properties in Table 1. [Computational fluctuations are smaller than half of the height of the represented points.] 
b)

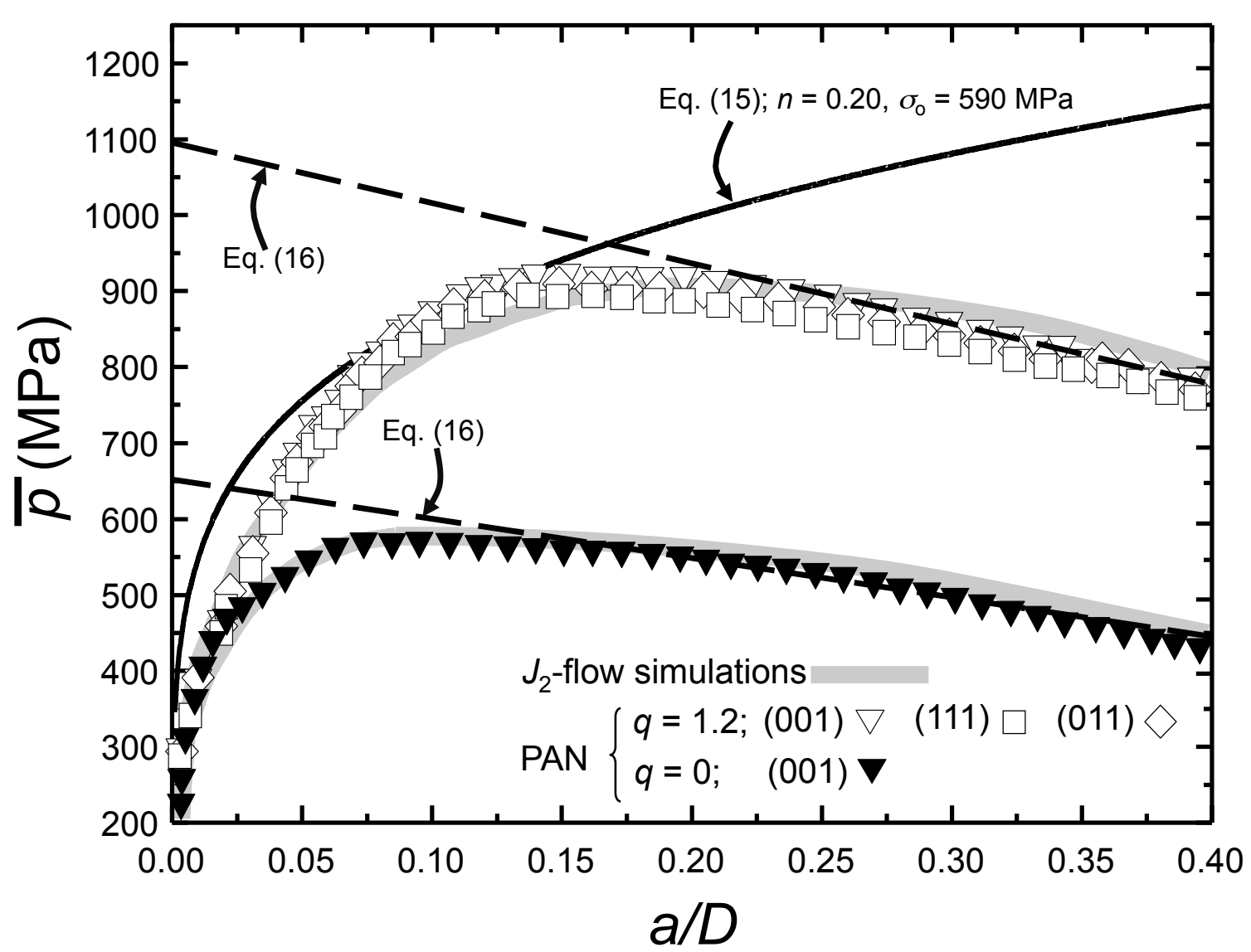

Fig. 3: Evolutions of hardness, $\bar{p}$, with increasing normalized contact radius, $a / D$. Part (a) is for pure copper (BW models), where the crystal plasticity simulations for the (001), (011) and (111) orientations overlap within computational scatter. The indentation behavior is archetypal of that in strong strain hardening crystals where stage-II dominates. Part (b) is for the PAN model where the gradual hardness decrease at $a / D>0.15$ is characteristic of the marked strain hardening saturation response during single-crystal stage-III. Notice that orientation-independent hardnesses are found in Parts (a) and (b). $J_{2}$-flow theory FE simulations are performed with the inferred properties in Table 1. [Computational fluctuations are smaller than half of the height of the represented points.] 
a)

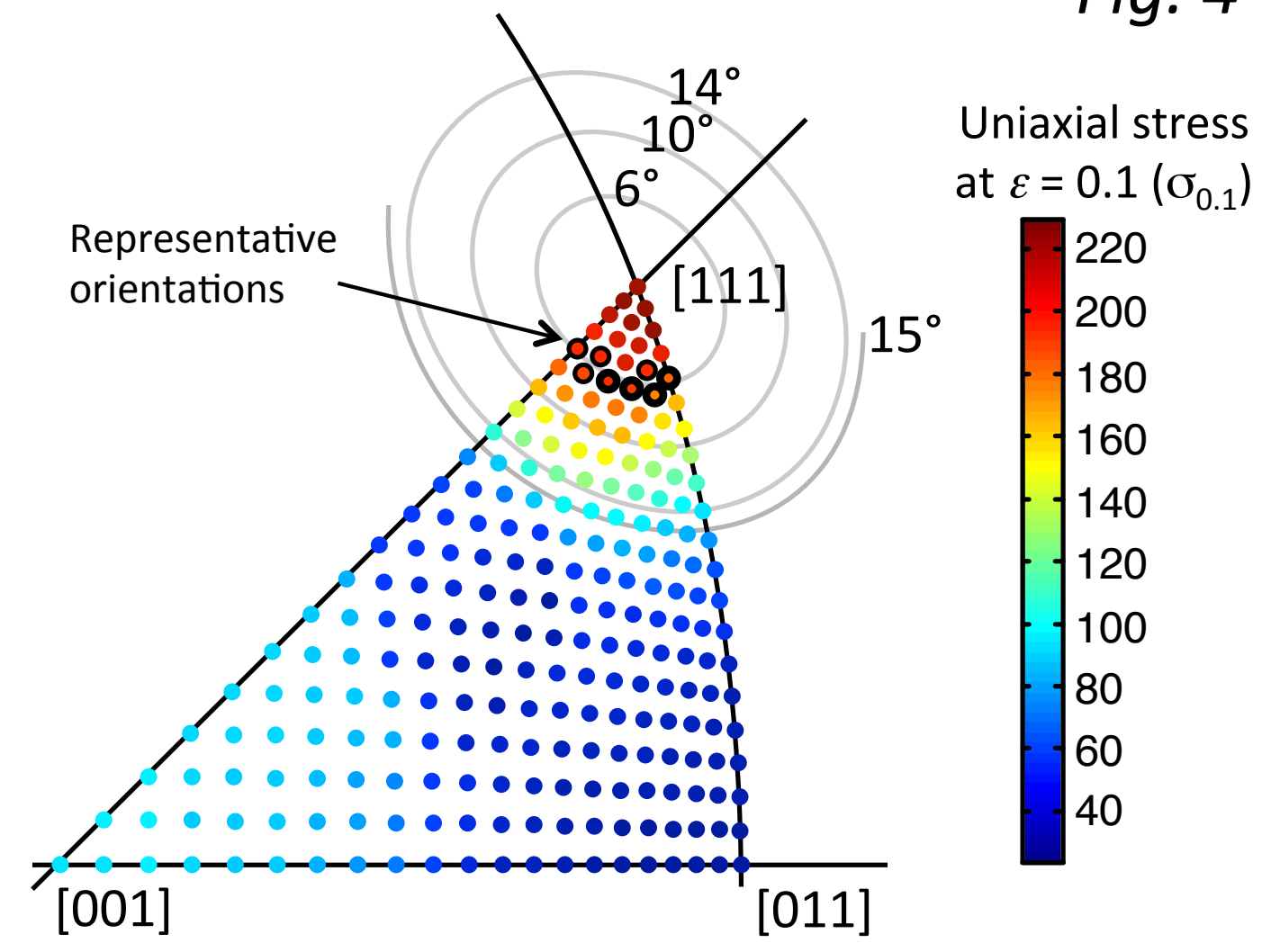

Fig. 4

b)

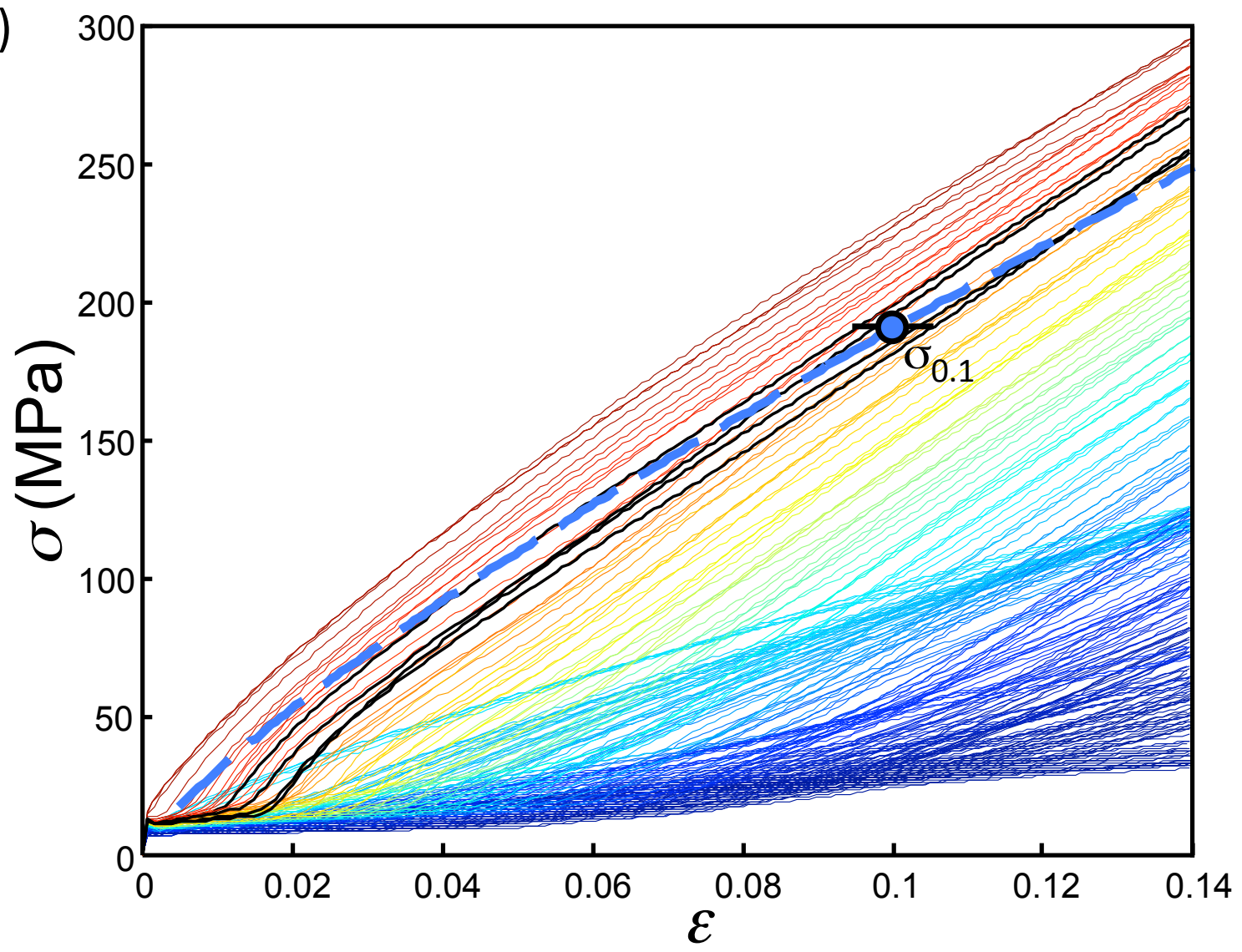

Fig. 4: Comparison between the single-crystal uniaxial response and the inferred stress-strain curve from indentation experiments. The results are for the modified BW model. Part (a) shows the azimuthal equal-area projection with the 5 to $7^{\circ}$ misorientations with respect to the [111] axis with similar $\sigma_{0.1}$ as that inferred from indentation (Table 3). Part (b) provides the uniaxial stress-strain curves with the same color code as Part (a). The thick discontinuous blue line in Part (b) is the inferred stress-strain curve from spherical indentation, where $\sigma_{0.1}$ is marked. Black continuous curves in Part (b) are for the specific representative misorientations highlighted with thick lines in Part (a). The stress-strain curves from these misorientations are in best agreement with the inferred uniaxial response throughout a wide range of strain levels. 
a)

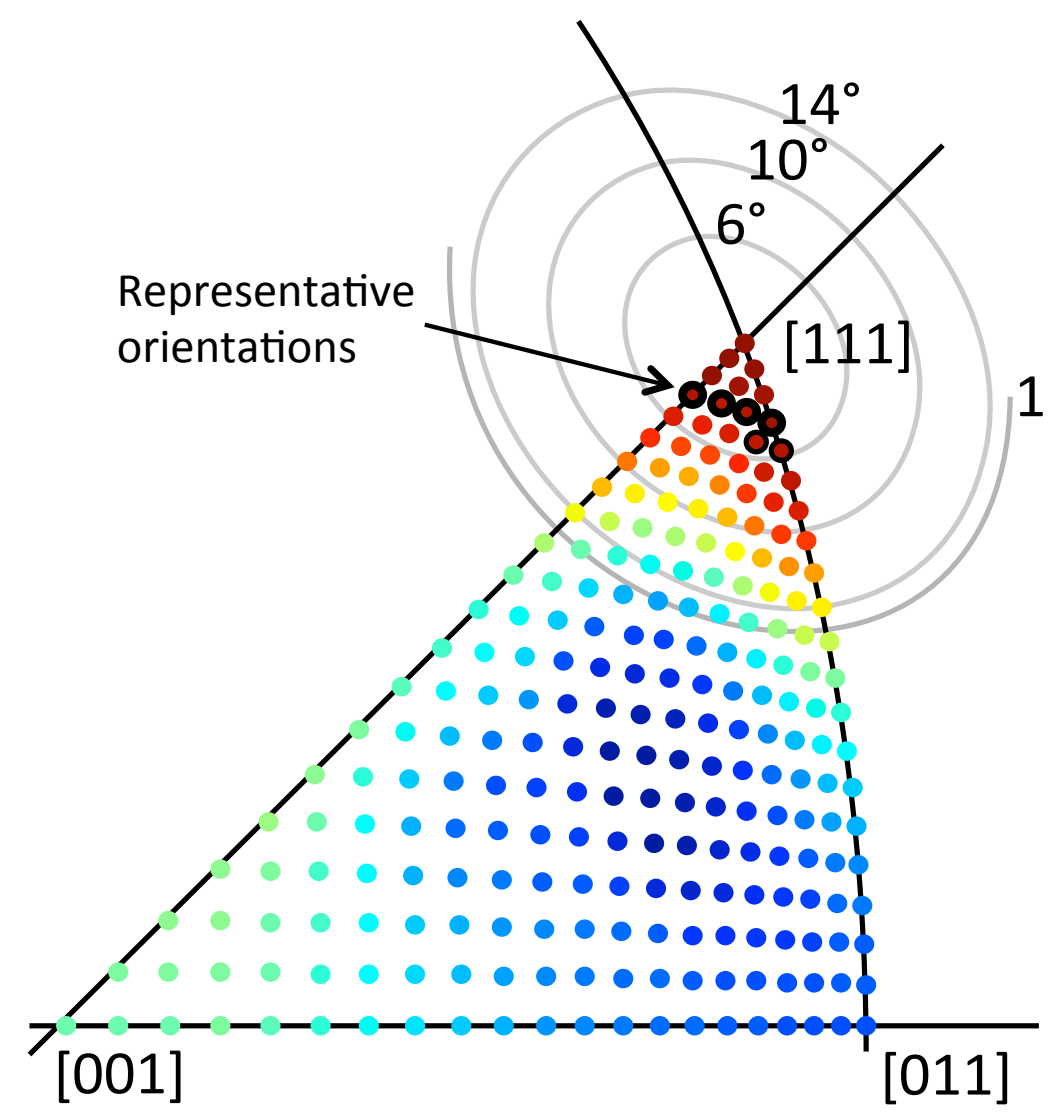

Fig. 5

\section{Uniaxial stress}

at $\varepsilon=0.1\left(\sigma_{0.1}\right)$

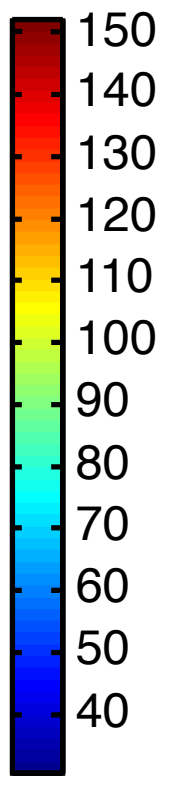

b)

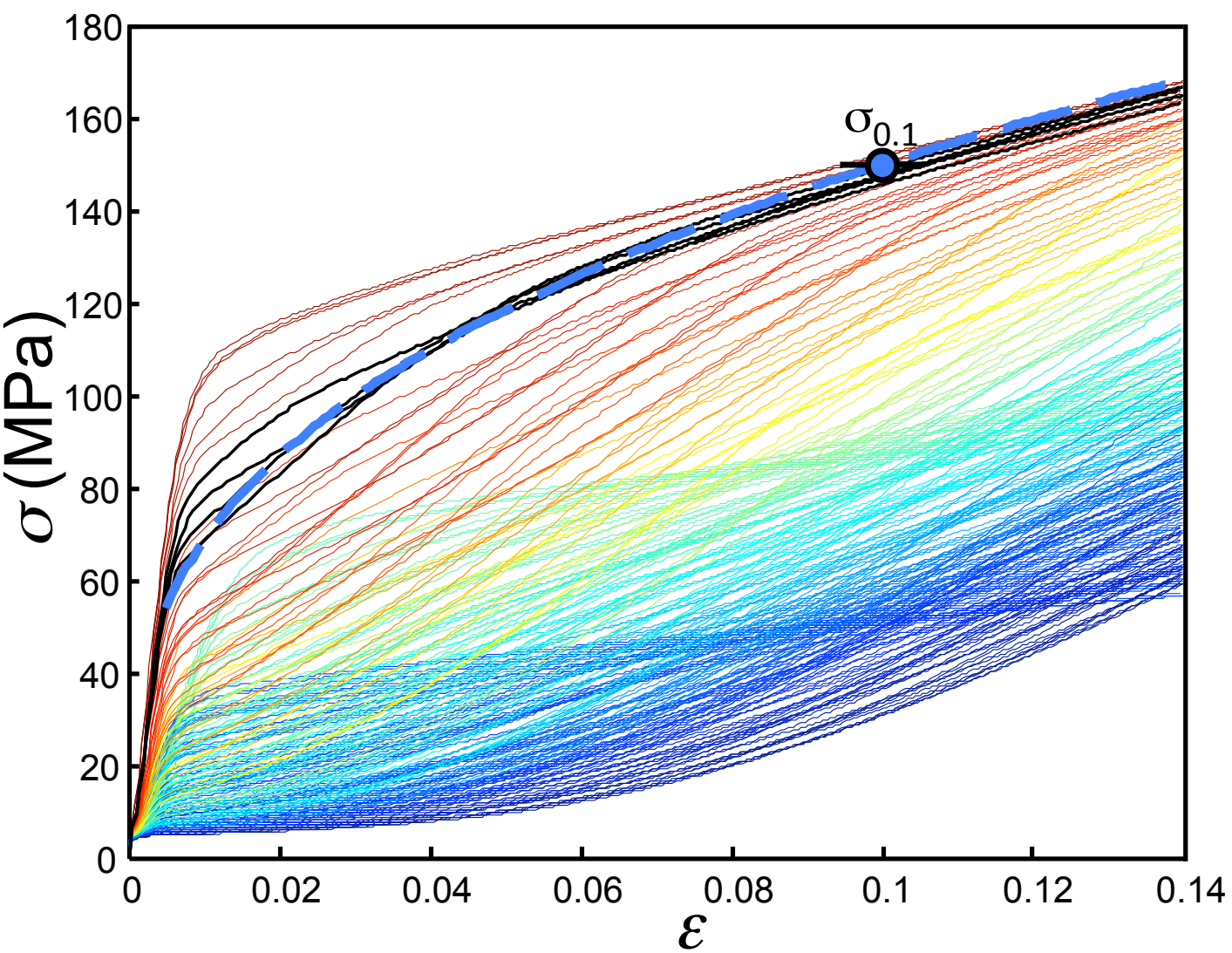

Fig. 5: Comparison between the single-crystal uniaxial response and the inferred stress-strain curve from indentation experiments. The results are for the original BW model. Part (a) shows the azimuthal equal-area projection with the 4 to $6^{\circ}$ misorientations with respect to the [111] axis with similar $\sigma_{0.1}$ as that inferred from indentation (Table 3). Part (b) provides the uniaxial stress-strain curves with the same color code as Part (a). The thick discontinuous blue line in Part (b) is the inferred stress-strain curve from spherical indentation, where $\sigma_{0.1}$ is marked. Black continuous curves in Part (b) are for the specific representative misorientations highlighted with thick lines in Part (a). The stress-strain curves from these misorientations are in best agreement with the inferred uniaxial response throughout a wide range of strain levels. 
a)

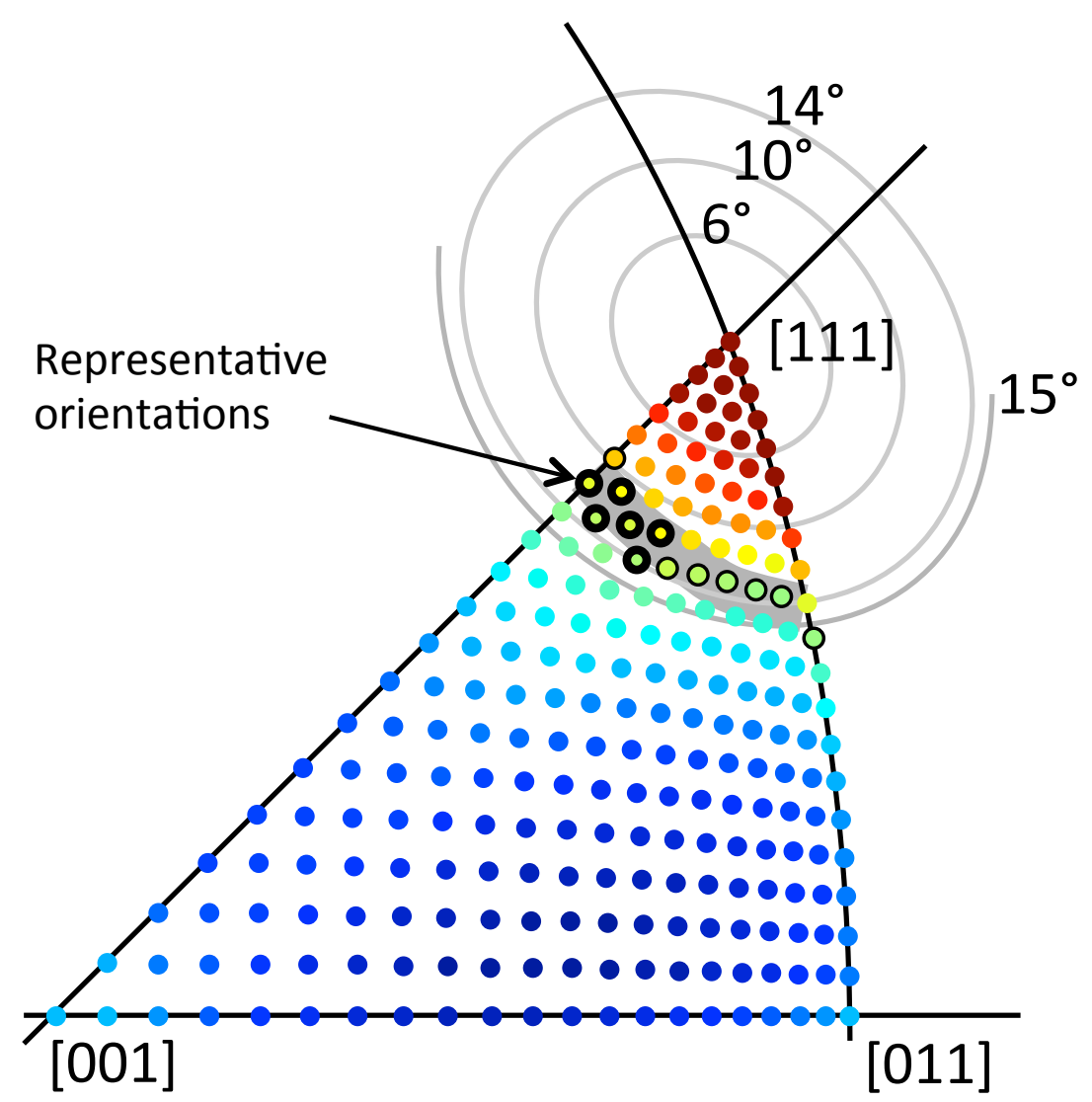

\section{Uniaxial stress}

at $\varepsilon=0.1\left(\sigma_{0.1}\right)$

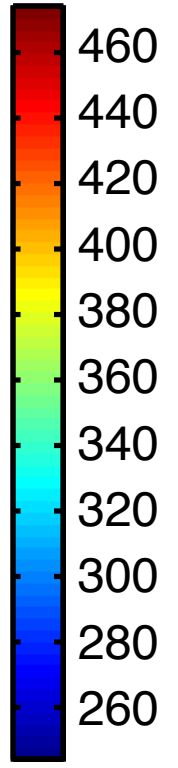

b)

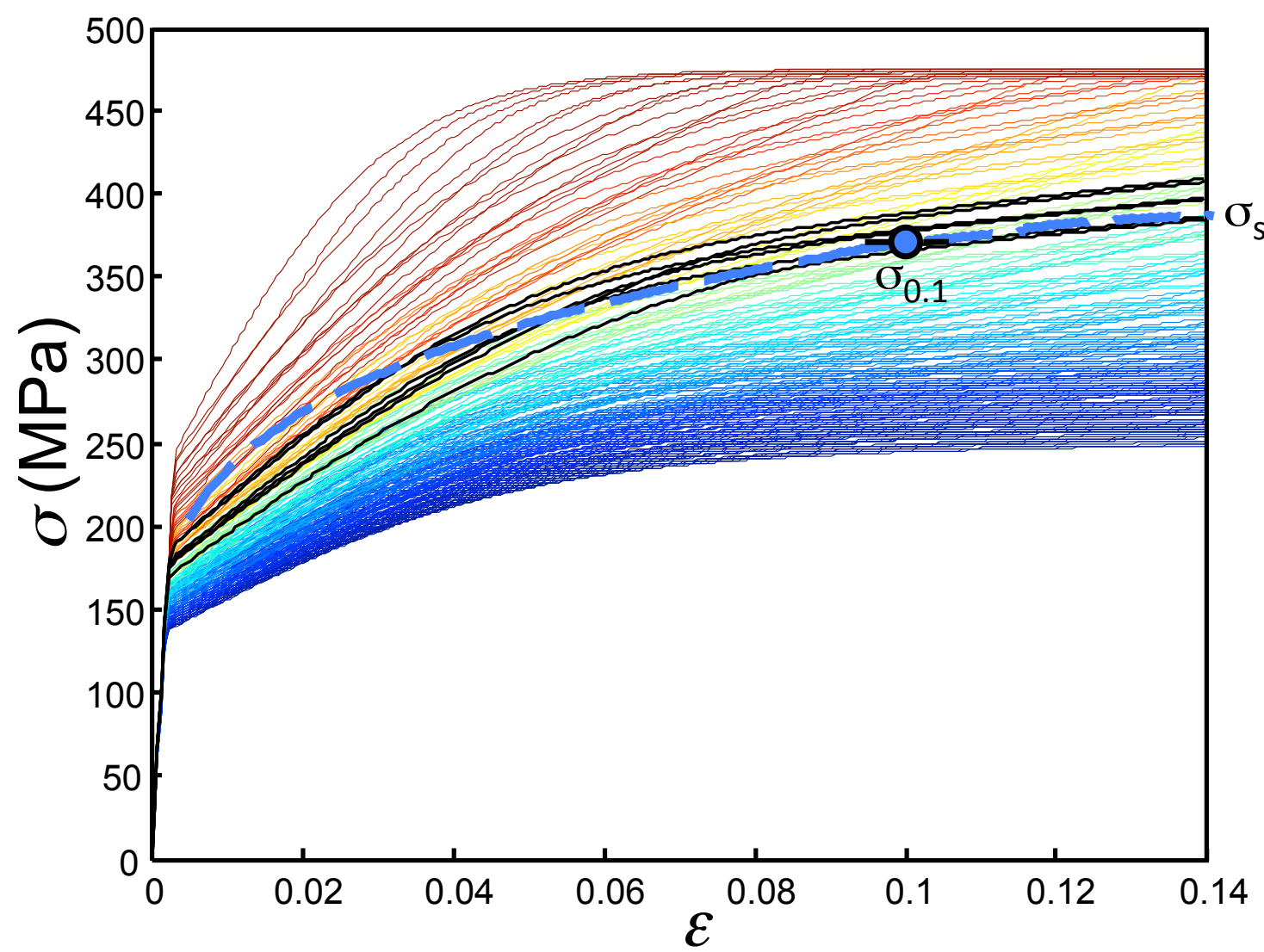

Fig. 6: Comparison between the single-crystal uniaxial response and the inferred stress-strain curve from indentation experiments. The results are for the PAN model at $q=1.2$. Part (a) shows the azimuthal equal-area projection with the 10 to $15^{\circ}$ misorientations with respect to the [111] axis with similar $\sigma_{0.1}$ as that inferred from indentation (Table 3). Part (b) provides the uniaxial stress-strain curves with the same color code as Part (a). The thick discontinuous blue line in Part (b) is the inferred stress-strain curve from spherical indentation, where $\sigma_{0.1}$ is marked. Black continuous curves in Part (b) are for the specific representative misorientations highlighted with thick lines in Part (a). The stress-strain curves from these misorientations are in best agreement with the inferred uniaxial response throughout a wide range of strain levels. 
a)

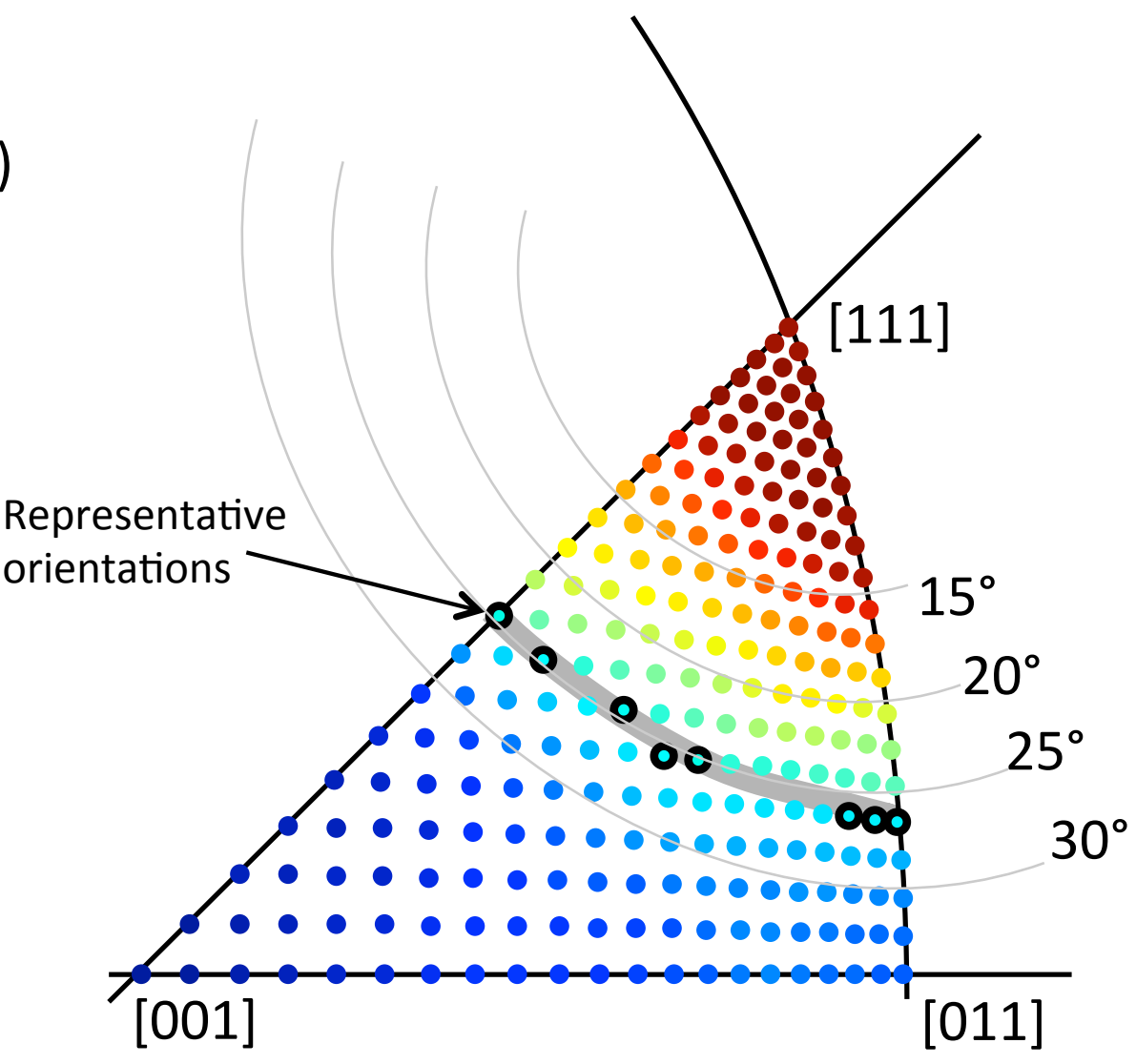

Uniaxial stress

at $\varepsilon=0.1\left(\sigma_{0.1}\right)$

270

260

250

240

230

220

210

200

190

180

b)

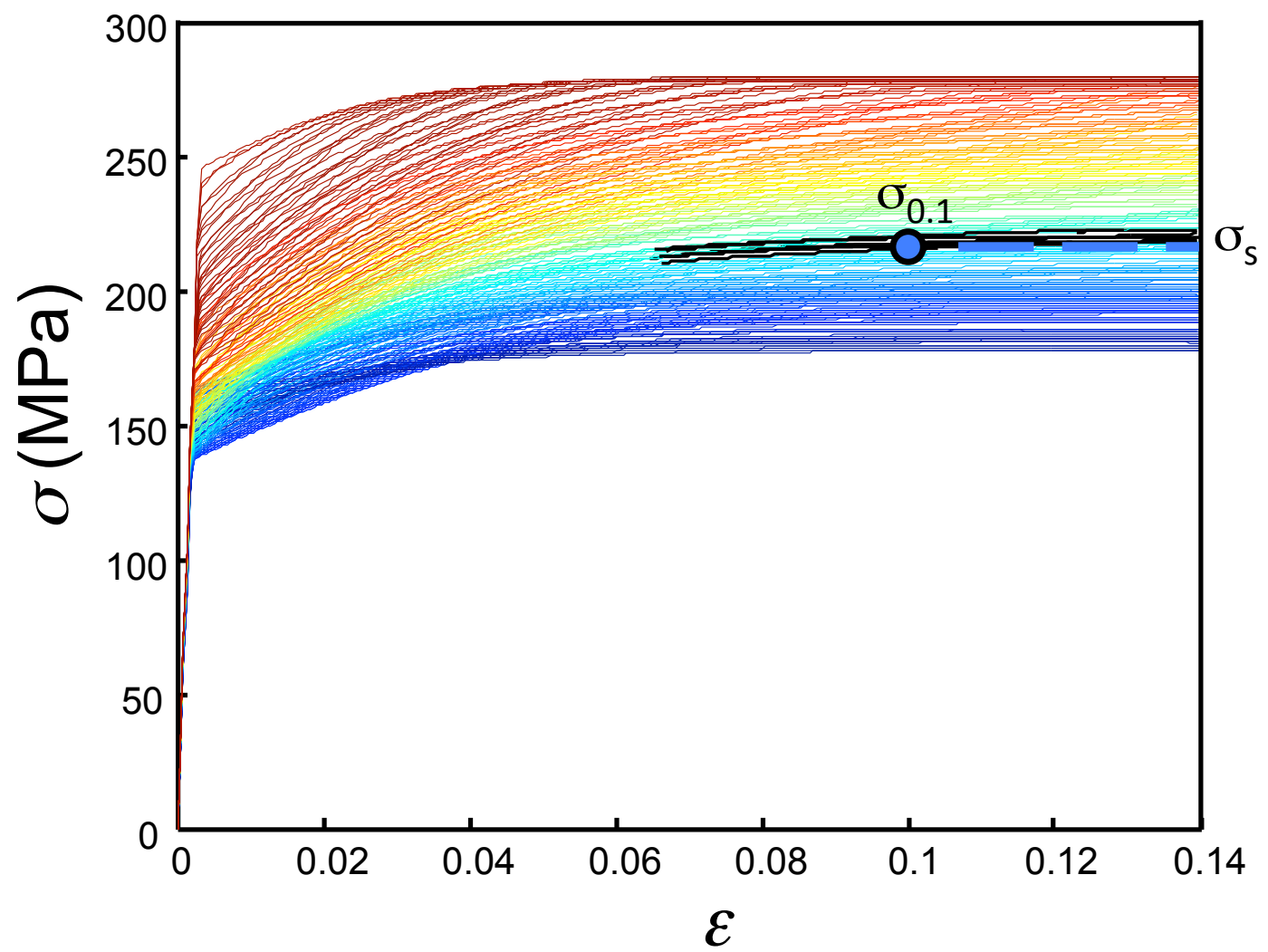

Fig. 7: Comparison between the single-crystal uniaxial response and the inferred stress-strain curve from indentation experiments. The results are for the PAN model at $q=0$ and concern the single crystal strain hardening saturation response. Part (a) shows the azimuthal equal-area projection with the 25 to $27^{\circ}$ misorientations with respect to the [111] axis (marked with thick black lines) with nearly identical saturation stress $\sigma_{s}$ as that inferred from indentation (Table 3). Part (b) provides the uniaxial stress-strain curves with the same color code as Part (a). The thick discontinuous line in Part (b) marks the value of $\sigma_{s}$ extracted from spherical indentation, which approximates to the value of $\sigma_{0.1}$ in the aforementioned misorientations. 
Table 1: Mechanical properties extracted from spherical indentations

\begin{tabular}{lcccccc}
\hline \hline & $\sigma_{\mathrm{o}}(\mathrm{MPa})$ & $n$ & $\sigma_{0.1}(\mathrm{MPa})^{1}$ & $\sigma_{\mathrm{s}}(\mathrm{MPa})$ & $\sigma_{\mathrm{ys}}(\mathrm{MPa})^{2}$ & $E(\mathrm{GPa})^{3}$ \\
\hline Original BW model & 341 & 0.34 & 150 & - & 17 & 110 \\
Modified BW model & 877 & 0.80 & 190 & - & $<1$ & 110 \\
PAN model $(q=1.2)$ & $590^{4}$ & $0.20^{4}$ & 370 & 360 & 179 & 70 \\
PAN model $(q=0)$ & $308^{5}$ & $0.10^{5}$ & 244 & 215 & 169 & 70 \\
\hline \hline
\end{tabular}

${ }^{1}$ Computed from Eq. (14): $\sigma_{0.1}=\sigma_{0}(0.1)^{n}$

${ }^{2}$ Computed from Eq. (15): $\sigma_{y s}=\left(\sigma_{0} / E^{n}\right)^{1 / 1-n}$

${ }^{3}$ Young's moduli are for copper $(E=110 \mathrm{GPa})$ and aluminum $(E=70 \mathrm{GPa})$ polycrystals

${ }^{4}$ From Eq. (16) for $0.07<a / D<0.13$

${ }^{4,5}$ From FlowChart1 in Alcalá and Esqué-de los Ojos (2010) $(a / D<0.13$ for $q=1.2$ and $a / D<0.08$ for $q=0)$

Table 2: Pyramidal (Berkovich) hardness and derived uniaxial stress $\sigma_{0.1}$

\begin{tabular}{llc}
\hline \hline & Hardness, $\bar{p}(\mathrm{MPa})^{1}$ & $\sigma_{0.1}(\mathrm{MPa})^{2}$ \\
\hline Original BW model & $450-480$ & $173-184$ \\
Modified BW model & $420-450$ & $162-173$ \\
PAN model $(q=1.2)$ & $860-880$ & $331-338$ \\
PAN model $(q=0)$ & $545-555$ & $210-215$ \\
\hline \hline
\end{tabular}

${ }^{1}$ Hardness range from the crystal-plasticity simulations for the different surface orientations.

${ }^{2}$ Computed from Eq. (18). 
Table 3: Orientation vectors where $\sigma_{0.1}$ coincides with the value inferred through indentation (see Figs. 4(a)-7(a))

\begin{tabular}{|c|c|}
\hline & Orientations in the azimuthal projection \\
\hline Original BW model & $\begin{array}{l}{\left[\begin{array}{lll}85 & 85 & 100\end{array}\right]^{1} ;\left[\begin{array}{lll}85 & 90 & 100\end{array}\right]^{1} ;\left[\begin{array}{lll}85 & 95 & 100\end{array}\right]^{1} ;\left[\begin{array}{ll}85 & 100 \\
100\end{array}\right]^{1} ;\left[\begin{array}{lll}80 & 95 & 100\end{array}\right]\left[\begin{array}{lll}80 & 100 & 100\end{array}\right]}\end{array}$ \\
\hline Modified BW model & 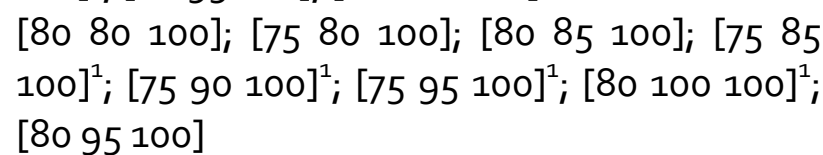 \\
\hline PAN model $(q=1.2)$ & 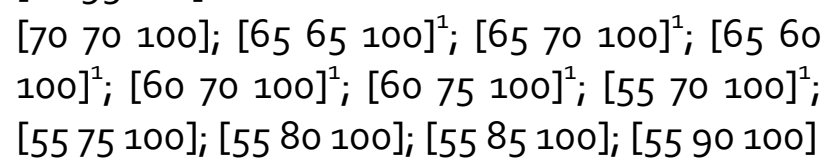 \\
\hline PAN model $(q=0)$ & 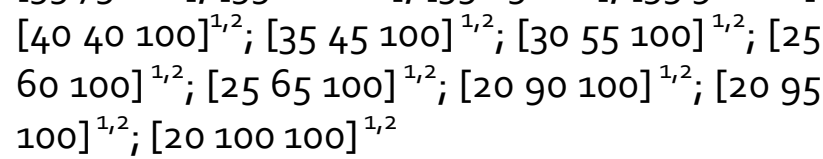 \\
\hline
\end{tabular}

${ }^{1}$ Orientations highlighted with black thick lines in Parts (a) from Figs. 4 to 7.

${ }^{2}$ In the selected orientations, $\sigma_{s}=\sigma_{0.1}$ 